\title{
Two new flavonoids and anticancer activity of Hymenosporum flavum: in vitro and molecular docking studies
}

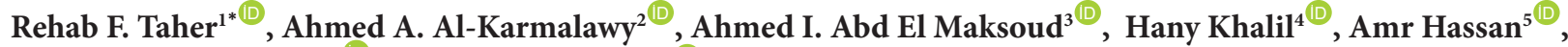 \\ Ezzel-Din A. El-Khrisy ${ }^{10}$, Walaa A. El-Kashak ${ }^{1 \mathbb{1}}$ \\ ${ }^{1}$ Chemistry of Natural Compounds Department, National Research Centre, 12622 Giza, Egypt \\ ${ }^{2}$ Department of Pharmaceutical Medicinal Chemistry, Faculty of Pharmacy, Horus University-Egypt, New Damietta 34518, Egypt \\ ${ }^{3}$ Industrial Biotechnology Department, Genetic Engineering and Biotechnology Research Institute, University of Sadat City, Sadat City, Egypt \\ ${ }^{4}$ Department of Molecular Biology, Genetic Engineering and Biotechnology Research Institute, University of Sadat City, Sadat City, Egypt \\ ${ }^{5}$ Bioinformatic Department, Genetic Engineering and Biotechnology Research Institute, University of Sadat City, Sadat City, Egypt
}

\section{A R T I C L E I N F O}

\section{Article Type:}

Original Article

\section{Article History:}

Received: 15 May 2021

Accepted: 23 July 2021

\section{Keywords:}

Anticancer activity

Flavonoids

Hymenosporum flavum

Molecular docking

Sorafenib

\begin{abstract}
A B S T RACT
Introduction: Hymenosporum flavum (Hook.) F. Muell. is the sole species within the genus Hymenosporum known for its antimicrobial activity. The current study aims to examine the prospective activity of $H$. flavum as a safe supporter of sorafenib (as a reference standard) against hepatocellular carcinoma (HCC).

Methods: Isolation and identification of compounds were made by chromatographic and spectroscopic methods. A fingerprint for the plant extract was done using HPLC-MS/MS spectrometric analysis. The total plant extract was examined in vitro for HCC activity. The isolated flavonoids were examined for their cytotoxic activities using molecular docking studies against both RAF-1 and ERK-2, and the promising compounds were further examined in vitro using quantitative reverse transcription polymerase chain reaction (qRT-PCR).

Results: Two new flavonols were isolated from the leaf extract of $H$. flavum (Hook.) F. Muell., quercetin-3-O-(glucopyranosyl $1 \rightarrow 2$ ribopyranoside) (1) and kaempferol-3-O-(glucopyranosyl $1 \rightarrow 2$ ribopyranoside) (2), accompanying other six known flavonoids (3-8), and identified via spectroscopic analysis. Moreover, HPLC- PDA/MS/MS spectrometric analysis revealed the presence of seventy phenolic metabolites. The cytotoxic activity of the plant extract confirmed its potential action on HepG2 cells indicated by the production level of lactate dehydrogenase (LDH) upon treatment compared with the normal cells. The isolated flavonoids were examined for their cytotoxic activity using molecular docking studies against both RAF-1 and ERK-2 as proposed mechanisms of their anticancer activities. Furthermore, compounds $\mathbf{1}$ and 3, which showed the best in silico results, were further examined in vitro using qRT-PCR. They exhibited promising inhibitory activities against both RAF-1 and ERK-2 gene expression. Moreover, they showed promising cytotoxic activities indicated by the MTT assay. Also, both of them improved the efficiency of sorafenib in targeting both RAF-1 and ERK-2 pathways suggesting synergistic combinations.

Conclusion: Our findings showed the potential cytotoxic activity of $H$. flavum extract on HepG2 cells. Some isolated compounds ( 1 \& 3 ) exhibited promising inhibitory activities against both RAF-1 and ERK-2 gene expression giving a lead future study for these compounds to be used in pharmaceutical preparations either alone or in combination with sorafenib.
\end{abstract}

Implication for health policy/practice/research/medical education:

Compounds isolated from the leaf extract of Hymenosporum flavum showed promising inhibitory activities against both RAF1 and ERK-2 gene expression. They also showed promising cytotoxic activities indicated by the MTT assay. Thus, the plant phytoconstituents could give a lead structure for drug development strategies against cancer.

Please cite this paper as: Taher RF, Al-Karmalawy AA, Abd El Maksoud AI, Khalil H, Hassan A, El-Khrisy EA, et al. Two new flavonoids and anticancer activity of Hymenosporum flavum: in vitro and molecular docking studies. J Herbmed Pharmacol. 2021;10(4):443-458. doi: 10.34172/jhp.2021.52. 


\section{Introduction}

Medicinal plants, since the days of yore, have been a promising source for natural drugs. Their safety aspects, low costs, and accessible approach gave them precedence to be investigated for their bioactive phytochemicals to be applied as natural medicines (1). Phytochemical component, mainly phenolics, have perpetually proved their importance as anticancer drugs (2).

Hymenosporum flavum (Hook.) F. Muell. is the only species within the genus Hymenosporum, closely related to the widespread genus Pittosporum (3). Several phenolics were previously isolated from the $H$. flavum leaves as quercetin-3-O- $\alpha$-L-rhamnopyranosyl$(1 \rightarrow 6)-\beta-D$-glucopyranoside, kaempferol-3-O- $\beta$-Dglucopyranosyl-( $1 \rightarrow 2)-\beta$-D-glucopyranoside, 3,5-di-Ocaffeoylquinic acid methyl ester, rosmarinic acid methyl ester, quercetin-3-O- $\alpha$-L-rhamnopyranoside, caffeic acid methyl ester, quercetin, and kaempferol. The ethyl acetate leaf extract was examined for its antimicrobial effect against six different bacterial strains (4). We reported the first fingerprint of bioactive compounds of $H$. flavum using high-performance liquid chromatography coupled with electrospray ionization tandem mass spectrometry (HPLC-ESI-MS/MS). Moreover, a part of our ongoing research was to isolate and determine the flavonoids content of the aqueous-alcoholic leaf extract.

Hepatocellular carcinoma (HCC) affects around one million persons worldwide every year (5). Hepatitis B virus (HBV) infections or mitogens with other tumor cell signaling cascades enhance RAF/MEK/ERK pathway. This pathway has a paramount role in poor prognostication and liver oncogenesis (6). Sorafenib is one of the targeted treatments, recognized as a kinase inhibitor and approved by the Food and Drug Administration (FDA) for HCC treatment (7).

RAF-1 has the leading role in the growth factor signal transduction from the cell membrane to the nucleus. Interestingly, RAF-1 kinase is activated and rapidly phosphorylated through stimulation by many mitogens (8). The mitogen-activated protein kinase (MAPK) pathway is considered the important signal transduction cascade that controls and regulates cell growth. The mechanism of MAPK activation occurs through the binding of growth factors to their cognate receptors. Also, it recruits the small GTPase Ras, which in sequence recruits the serine-threonine kinase RAF-1 (9). MAPK has many pathways that control several cellular processes, differentiation, and drives proliferation and cell survival. One of these pathways is ERK (extracellular-signalregulated kinase) signaling (10).

Numerous researchers established a fruitful combination between sorafenib and other compounds targeting signaling pathways for improving its efficacy. Sorafenib combined with OSU-2S with a synergistic effect to boost the antiproliferative activity on HCC cells, where tumor protein p53 and Protein kinase C-delta (PKC $\delta$ ) are associated with the regulation of OSU-2S/sorafenibinduced cell death (11). Numerous studies have shown the synergistic effect of sorafenib alongside various active compounds in plant extracts as resveratrol in grapes, peanuts, red wine (12), corosolic acid in Actinidia chinensis (13), and wogonin (5,7-dihydroxy-8-methoxyflavone) in Scutellaria baicalensis (14).

For the time being, new therapeutics discovery or repurposing already existing ones become faster by using several computational methods as molecular docking. Molecular docking is of paramount importance to propose the expected mechanisms of action for the tested compounds and their repurposing (15). Take into consideration the essential role of both RAF-1 and ERK-2 pathways for cancer development. Besides the previously reported effects of anticancer drugs targeting these genes (16), and in continuation to our previous work targeting cancer (17), our goal in this research was to investigate the cytotoxic activity of $H$. flavum hydroalcoholic leaf extract against human liver tumor cells. We investigated the possible synergistic combinations of the most promising compounds of the leaf extract with sorafenib through RAF/MEK/ERK signaling pathways inhibition, using in silico and in vitro studies. Meanwhile, the fingerprint profile of the leaf extract, the determination of isolated compounds, and their anticancer activities could supply beneficial information on the importance of the plant and derived natural remedies. Collectively, the present study consequences a novel formula of $H$. flavum hydroalcoholic leaf extract to be used in HCC therapeutic protocols.

\section{Materials and Methods}

General

NMR spectra were recorded on Bruker Avance III $400 \mathrm{MHz}$ for ${ }^{1} \mathrm{H}$ and $100 \mathrm{MHz}$ for ${ }^{13} \mathrm{C}$ (Bruker AG, Switzerland) with BBFO Smart Probe and Bruker 400 AEON Nitrogen-Free Magnet. Data were analyzed using Topspin 3.1 software. Stationary phases used for column chromatography were normal phase polyamide (Sigma-Aldrich Chemicals) and Sephadex LH-20 (E Merck).

Plant materials

Hymenosporum flavum leaves were collected from Mostafa Al Abd-Farm, Alexandria Desert Road, Egypt, in March 2015 and kindly authenticated by Dr. Mohamed El-Gebali, Department of Botany, National Research Centre. Voucher specimens (2015-13) were deposited in the Herbarium of the National Research Centre.

\section{Extract preparation}

The powder of air-dried leaves of $H$. flavum $(1.2 \mathrm{~kg})$ was extracted with $\mathrm{CH}_{3} \mathrm{OH}: \mathrm{H}_{2} \mathrm{O}[7: 3(6 \times 3 \mathrm{~L})]$ at room temperature. The extract was filtered and evaporated under reduced pressure to give a dry end product (150 
$\mathrm{g}$ ), redissolved in $500 \mathrm{~mL}$ water for further chemical and biological investigation.

\section{HPLC- ESI-MS/MS analysis}

\section{Sample preparation}

We prepared the sample $(120 \mu \mathrm{g} / \mathrm{mL})$ solution using HPLC analytical grade solvent of $\mathrm{MeOH}$, filtered the sample using a membrane disc-filter $(0.2 \mu \mathrm{m})$, then subjected it to LC-ESI-MS analysis.

\section{Apparatus and conditions}

Using HPLC- ESI-MS/MS, the aqueous methanolic extract of $H$. flavum was analyzed. The LC system was Thermo Finnigan (Thermo Electron Corporation, Austin, TX, USA) (18). The reversed-phase column Zorbax Eclipse XDB-C18, with rapid resolution, $4.6 \times 150 \mathrm{~mm}, 3.5 \mu \mathrm{m}$ column was used (Agilent, Santa Clara, CA, USA). The mobile phase was water and acetonitrile (ACN) $(0.1 \%$ formic acid each), and the gradient was employed from $5 \%$ to $30 \%$ in 60 minutes with a flow rate of $1 \mathrm{~mL} / \mathrm{min}$ with a 1:1 split before the ESI source. The samples were injected using an autosampler. LCQ-Duo ion trap with a Thermo Quest ESI source was used for MS analysis. We used Xcalibur software (Xcalibur ${ }^{\mathrm{TM}}$ 2.0.7, Thermo Scientific, Waltham, MA, USA) to control the system.

\section{Isolation of compounds}

Three hundred and fifty $\mathrm{ml}$ of water extract was loaded on a polyamide 6 column chromatography. The column was eluted with $\mathrm{H}_{2} \mathrm{O}$, and then the mixtures of $\mathrm{H}_{2} \mathrm{O}-\mathrm{MeOH}$ of decreased polarity to collect ten fractions $(1 \mathrm{~L}$, each). The main phenolic fractions obtained were united into six after chromatographic analysis via paper chromatography.

Fraction I (4.1 g) was fractionated by column chromatography on Sephadex $\mathrm{LH}-20$ with $\mathrm{H}_{2} \mathrm{O}$, followed by mixtures of $\mathrm{H}_{2} \mathrm{O}-\mathrm{EtOH}(0-80 \%)$ for elution to give compounds $1(28 \mathrm{mg})$ and $3(23 \mathrm{mg})$. Fraction II $(3.4 \mathrm{~g})$ was subjected to sub fractionation on Sephadex LH-20 with $\mathrm{H}_{2} \mathrm{O}$, followed by mixtures of $\mathrm{H}_{2} \mathrm{O}-\mathrm{EtOH}(0-70 \%)$ for elution to give pure compounds $2(20 \mathrm{mg})$ and 4 (18 $\mathrm{mg})$. Using the same procedure, fraction III (3 g) gave chromatographically pure samples $5(13 \mathrm{mg})$ and $6(15$ $\mathrm{mg})$. Compounds $7(14 \mathrm{mg})$ and $8(17 \mathrm{mg})$ were purified from fraction $\mathrm{V}(4.5 \mathrm{~g})$ after subjection to Sephadex LH-20 column and eluting by $\mathrm{H}_{2} \mathrm{O}-\mathrm{EtOH}(0-60 \%)$. We identified all eight compounds through spectroscopic methods $\left({ }^{1} \mathrm{H}\right.$, ${ }^{13} \mathrm{C}$ NMR, ESI-MS) compared with the literature.

\section{Docking studies}

Two separate molecular docking studies were performed through MOE 2019.012 suite (24) to propose, quantify, and evaluate the binding scores and interactions of the eight isolated flavonoids of $H$. flavum leaf extract against RAF1 and ERK-2 proteins, respectively. The co-crystallized inhibitors of both proteins were involved in our studies as reference standards.
Preparation of the isolated flavonoids from $\mathrm{H}$. flavum leaf extract

The examined isolated flavonoids (1-8) were sketched using MOE builder, adjusted for their partial charges, and then energy minimized to be ready for docking processes as previously discussed (25). Two different databases were built containing the prepared flavonoids (1-8), besides the co-crystallized inhibitor of RAF-1 ((1E)-5-(1-piperidine4-yl-3-pyridine-4-yl-1H-pyrazole-4-yl)-2,3-dihydro- $1 \mathrm{H}$ inden-1-one oxime) in one database and the co-crystallized one of ERK-2 ((1aR,8S,13S,14S,15aR)-5,13,14-trihydroxy3-methoxy-8-methyl-8,9,13,14,15,15a-hexah-ydro$6 \mathrm{H}$-oxireno[k][2]benzox-acyclotetradecine-6,12(1aH) -dione, hypothemycin) in the other database. We saved both databases as MDB files for the docking processes.

\section{Preparation of both RAF-1 and ERK-2 protein pockets}

The X-ray structures of c-RAF (RAF-1) (code: 3OMV) (26) and ERK-2 (code: 3C9W) (27) were downloaded from the Protein Data Bank, corrected, 3 D protonated, and prepared for docking following the detailed preparation steps described earlier (28).

Docking of the isolated extract flavonoids to the binding pockets of RAF-1 and ERK-2

Following the general docking methodology applied before, the two mentioned databases were docked in two separate docking processes (29). Finally, we selected the best pose for each docked compound at each target receptor according to scores, RMSD-refine values, and binding interactions for further studies. Also, we performed a program validation at first for both targets by redocking the co-crystallized inhibitor in each case, and we approved the validity by low RMSD values $(<1)$ between the docked and native forms (10).

\section{In vitro studies}

\section{Cell line}

Hepatocellular carcinoma (HepG2 cells) was grown in RPMI media supplemented with $4 \mathrm{mM}$ L-glutamine, $4 \mathrm{mM}$ sodium pyruvate, and $2.5 \%$ heat-treated bovine serum albumin (BSA). The normal hepatocyte cells were grown in RPMI media containing $4 \mathrm{mM} \mathrm{L}$-glutamine and $10 \%$ BSA. We incubated all cell lines at $37^{\circ} \mathrm{C}$ under $5 \%$ $\mathrm{CO}_{2}$ condition (30). The imaging of cultured cells was determined by using inverted microscopy with a Zeiss A-Plan 10X.

\section{Cytotoxic concentration $50 \%\left(C C_{50}\right)$}

We tested the purified agent for its cytotoxic effect and calculated the potential $\mathrm{CC}_{50}$ on HepG2 cells and the normal hepatocytes. Therefore, the cells were cultured in 96-well plates in a density of $10 \times 10^{3}$ cells/well and incubated in a $\mathrm{CO}_{2}$ incubator at $37^{\circ} \mathrm{C}$. The cells were treated with different concentrations of the purified agent 
(0-25 $\mathrm{mg} / \mathrm{mL})$ followed by overnight incubation. The cell viability rate and the cytotoxic concentration were monitored using an MTT cell growth assay kit (SigmaAldrich, Germany). Based on the amount of formazan dye, the $\mathrm{CC}_{50}$ was measured by measuring the absorbance at $570 \mathrm{~nm}$.

\section{Lactate dehydrogenase ( $\mathrm{LDH}$ ) production}

We used the LDH assay kit (Abc-65393) to assess LDH production in the medium collected from culturedtreated cells. According to the manufacturing procedures, $100 \mu \mathrm{L}$ of lysed cells was incubated with a $100 \mu \mathrm{L} \mathrm{LDH}$ reaction mix for 30 minutes at room temperature. $\mathrm{LDH}$ activity was quantified by a plate reader at OD450 $\mathrm{nm}$. The relative $\mathrm{LDH}$ production was calculated by dividing the mean values of the treated cells on the mock values, which indicated by fold change (31).

\section{Reverse transcription and quantitative real time-PCR}

HepG2 cells were seeded at a density of $2 \times 10^{5}$ cells per well in a six-well plate followed by overnight incubation. The cells were treated with $100 \mu \mathrm{g} / \mathrm{mL}$ of each purified flavonoid and (or) SOR followed by incubation at $37^{\circ} \mathrm{C}$ in a $\mathrm{CO}_{2}$ incubator for 24 hours. To quantify messenger RNA (mRNA) of indicated genes, we used quantitative reverse transcription polymerase chain reaction (qRT-PCR) to make cDNA construction and amplification in one step via the purified total RNA as a template. Total RNA was extracted 24 hours post-treatment from treated cells and purified using the RNeasy Mini Kit (Qiagen, USA) and TriZol (Invitrogen, USA). The relative expressions of Raf-1 and Erk-2 were detected with the QuantiTect SYBR Green PCR Kit (Qiagen, USA) and oligonucleotides specific for each gene (Table 1). Housekeeping glyceraldehyde 3-phosphate (GAPDH) gene level was used for normalization. The following mixture was prepared for each reaction: $10 \mu \mathrm{L}$ SYBR Green, $0.5 \mu \mathrm{L}$ reverse transcriptase $(50 \mathrm{U} / \mu \mathrm{L}), 0.2 \mu \mathrm{L}$ RNase inhibitor $(20 \mathrm{U} /$ $\mu \mathrm{L}), 1 \mu \mathrm{L}$ purified total RNA $(100 \mathrm{ng} / \mu \mathrm{L})$, and $1 \mu \mathrm{L}$ from each primer up to a final volume of $25 \mu \mathrm{L}$ using RNase free water. According to the manufacturer's protocol, we used the following PCR parameters: $50^{\circ} \mathrm{C}$ for 30 minutes, $95^{\circ} \mathrm{C}$ for 3 minutes, 35 cycles $\left(95^{\circ} \mathrm{C}\right.$ for $30 \mathrm{~s}, 60^{\circ} \mathrm{C}$ for $15 \mathrm{~s}, 72^{\circ} \mathrm{C}$ for $30 \mathrm{~s}$ ). We obtained levels of Raf-1 and Erk-2 relative to GAPDH using comparative $\Delta \Delta \mathrm{Ct}$ equations (16).

Table 1. Oligonucleotides sequence used for detection of steady-state mRNA of the indicated genes

\begin{tabular}{ll}
\hline Raf-1 sense & 5'-TTTCCTGGATCATGTTCCCCT-3' \\
\hline Raf-1 antisense & 5'-ACTTTGGTGCTACAGTGCTCA-3' \\
\hline ERK sense & 5'-TGTGCTCTGCTTATGATAATG-3' \\
\hline ERK antisense & 5'-GATGGTTGGTGCTCGAATAAT3' \\
\hline GAPDH-sense & 5'-TGGCATTGTGGAAGGGCTCA3' \\
\hline GAPDH-antisense & 5'-TGGATGCAGGGATGATGTTCT3' \\
\hline
\end{tabular}

Statistical analysis

The Microsoft Excel software was used for all histograms and chart preparations. For statistical analysis of the data, we used the Student's two-tailed $t$ test. $P$ values $\leq 0.05$ were considered statistically significant. $\Delta \Delta \mathrm{Ct}$ analysis was used to determine the mRNA expression fold change detected by qRT-PCR using the following equation: (1) $\Delta \mathrm{Ct}=\mathrm{Ct}$ value for gene- Ct value for GAPDH, (2) $(\Delta \Delta \mathrm{Ct})=1 \mathrm{Ct}$ for value (experimental) $-\Delta \mathrm{Ct}$ for value (control), Expression fold change $=(2-\Delta \Delta \mathrm{ct})(32)$.

\section{Results}

Isolation and identification of the compounds

Eight phenolic compounds were isolated from the hydroalcoholic leaf extract of $H$. flavum and identified using spectroscopic techniques.

Compound 1: Quercetin-3-O-(glucopyranosyl $1 \rightarrow 2$ ribopyranoside): obtained as fine yellow powder. Mp: 236$239^{\circ} \mathrm{C}$; HR-ESI-MS showing a molecular ion peak at [M$\mathrm{H}]^{-} \mathrm{m} / \mathrm{z} 595.3771,{ }^{1} \mathrm{H}$ and ${ }^{13} \mathrm{C}$ NMR data are represented in Figure 1 (chemical structures of compound) and $3\left({ }^{1} \mathrm{H}\right.$ and ${ }^{13} \mathrm{C}$ NMR of compounds).

Compound 2: Kaempferol-3-O-(glucopyranosyl $1 \rightarrow 2$ ribopyranoside): obtained as fine yellow needles. Mp: 234$237^{\circ} \mathrm{C}$; HR-ESI-MS showed a molecular ion peak at $[\mathrm{M}-\mathrm{H}]$ $\mathrm{m} / \mathrm{z}$ 579.3777; ${ }^{1} \mathrm{H}$ and ${ }^{13} \mathrm{C} \mathrm{NMR}$ data are represented in Figure 1 (chemical structures of compounds) and $3\left({ }^{1} \mathrm{H}\right.$ and ${ }^{13} \mathrm{C}$ NMR of compounds)

Compound 3: Quercetin-3-O- $\left(2{ }^{\prime \prime} \mathrm{O}-\beta-\mathrm{D}-\right.$ glucopyranosyl)- $\beta$-D-galactopyranoside: obtained as a yellow amorphous powder. ${ }^{1} \mathrm{H}$ and ${ }^{13} \mathrm{C}$ NMR data are represented in Table 2.

Compound 4: Quercetin-3-O-glucopyranoside: obtained as fine yellow powder. ${ }^{1} \mathrm{H}$ NMR (MeOD, 400 $\mathrm{MHz}): \delta 7.42\left(\mathrm{lH}, \mathrm{dd} . J=2.1 \mathrm{~Hz}\right.$ and $\left.8.4 \mathrm{~Hz}, \mathrm{H}-6^{\prime}\right) ; 7.40$ $\left(\mathrm{lH}, \mathrm{d}, J=2.1 \mathrm{~Hz}, \mathrm{H}-2^{\prime}\right) ; 6.79\left(1 \mathrm{H}, \mathrm{d}, J=8.4 \mathrm{~Hz}, \mathrm{H}-5^{\prime}\right) ; 6.31$ (l H, d, J=2.04 Hz, H-8); 6.12 (1H, d, J=2.04 Hz, H-6); 5.09 (lH, d, $J=7.5 \mathrm{~Hz}, \mathrm{H}-1{ }^{\prime \prime}$ ); ${ }^{13} \mathrm{CNMR}$ (MeOD, $100 \mathrm{MHz}$ ): 178.19 (C-4), 165.38 (C-7), 164.76 (C-5), 159.88 (C-9), 157.98 (C-2), 150.05 (C-4'), 148.49 (C-3'), 134.68 (C-3), $125.77\left(\mathrm{C}-6^{\prime}\right), 121.58\left(\mathrm{C}-1^{\prime}\right), 119.09\left(\mathrm{C}-2^{\prime}\right), 116.53\left(\mathrm{C}-5^{\prime}\right)$, 104.31 (C-10), 101.31 (C-6), 98.60 (C-8), 78.22 (C-5' '),

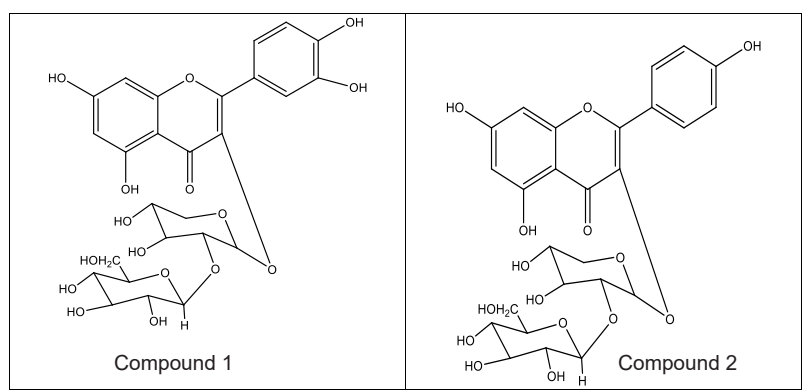

Figure 1. Chemical structures of compounds 1 and 2. 
Table 2. ${ }^{1} \mathrm{H}$ and ${ }^{13} \mathrm{C}$ NMR of compounds 1,2 \& 3 from $\mathrm{H}$. flavum extract

\begin{tabular}{|c|c|c|c|c|c|c|}
\hline & Compound 1 & & Compound 2 & & Compound 3 & \\
\hline Pos. & $\delta^{1} \mathrm{H}$ & $\delta^{13} \mathrm{C}$ & $\delta^{1} \mathrm{H}$ & $\delta^{13} \mathrm{C}$ & $\delta^{1} \mathrm{H}$ & $\delta^{13} \mathrm{C}$ \\
\hline \multicolumn{7}{|l|}{1} \\
\hline 2 & & 157.07 & & 155.16 & & 154.93 \\
\hline 3 & & 134.33 & & 132.72 & & 133.70 \\
\hline 4 & & 178.30 & & 178.67 & & 178.50 \\
\hline 5 & & 161.67 & & 161.67 & & 161.73 \\
\hline 6 & $6.23 \mathrm{~d}, J=2$ & 100.05 & $6.11 \mathrm{~d}, J=2.2$ & 99.86 & $6.10 \mathrm{~d}, J=2$ & 98.43 \\
\hline 7 & & 164.57 & & 164.25 & & 164.56 \\
\hline 8 & $6.41 \mathrm{~d}, J=2$ & 93.33 & $6.31 \mathrm{~d}, J=2.2$ & 93.35 & $6.29 \mathrm{~d}, J=2$ & 93.24 \\
\hline 9 & & 157.57 & & 157.09 & & 157.08 \\
\hline 10 & & 104.04 & & 103.39 & & 103.66 \\
\hline $1^{\prime}$ & & 121.46 & & 121.18 & & 121.46 \\
\hline $2^{\prime}$ & $7.63 d, J=2.04$ & 114.98 & $7.92 \mathrm{~d}, J=8.76$ & 130.97 & $7.64 \mathrm{~d}, J=2.1$ & 114.81 \\
\hline $3^{\prime}$ & & 144.75 & $6.83 \mathrm{~d}, J=8.76$ & 115.06 & & 144.52 \\
\hline $4^{\prime}$ & & 148.54 & & 160.24 & & 148.49 \\
\hline $5^{\prime}$ & $6.94 \mathrm{~d}, J=8.4$ & 116.01 & $6.83 \mathrm{~d}, J=8.76$ & 115.06 & $6.78 \mathrm{~d}, J=8.4$ & 116.40 \\
\hline $6^{\prime}$ & $7.62 \mathrm{dd}, J=2.04,8.4$ & 121.87 & $7.92 \mathrm{~d}, J=8.76$ & 130.97 & $7.46 \mathrm{dd}, J=2.1,8.4$ & 121.63 \\
\hline $1^{\prime \prime}$ & $5.41 \mathrm{~d}, J=3.8$ & 98.49 & $5.38, d, J=3.36$ & 98.51 & $5.16 \mathrm{~d}, J=7.6$ & 100.33 \\
\hline $2^{\prime \prime}$ & & 78.95 & $4.11, m$ & 78.61 & & 79.20 \\
\hline $3^{\prime \prime}$ & & 70.23 & \multirow{3}{*}{$\begin{array}{l}\text { In range } \\
(3.86-3.67)\end{array}$} & 69.96 & & 73.47 \\
\hline $4^{\prime \prime}$ & & 65.48 & & 67.73 & & 68.68 \\
\hline $5^{\prime \prime}$ & & 73.79 & & 73.79 & & 75.59 \\
\hline $6^{\prime \prime}$ & & 61.01 & & 61.23 & & 60.48 \\
\hline $1^{\prime \prime \prime}$ & $4.57 \mathrm{~d}, J=7.76$ & 104.34 & $4.46 \mathrm{~d}, J=7.72$ & 104.38 & $4.65 \mathrm{~d}, J=7$ & 104.29 \\
\hline $2^{\prime \prime \prime}$ & & 73.42 & $3.17 m$ & 73.47 & & 74.04 \\
\hline $3^{\prime \prime \prime}$ & & 76.52 & \multirow{4}{*}{$\begin{array}{l}\text { In range } \\
(3.23-3.11)\end{array}$} & 76.63 & & 76.66 \\
\hline $4^{\prime \prime \prime}$ & & 69.68 & & 69.89 & & 69.62 \\
\hline $5^{\prime \prime \prime}$ & & 76.52 & & 76.58 & & 76.50 \\
\hline $66^{\prime \prime \prime}$ & & 62.39 & & 65.27 & & 60.85 \\
\hline
\end{tabular}

$76.56\left(\mathrm{C}-3^{\prime \prime}\right), 73.98\left(\mathrm{C}-2^{\prime \prime}\right), 71.89\left(\mathrm{C}-4^{\prime \prime}\right), 62.97\left(\mathrm{C}-6^{\prime \prime}\right)$ (19).

Compound 5: Quercetin-3-O-galactopyranoside: obtained as fine yellow powder. ${ }^{1} \mathrm{H}$ NMR (MeOD, 400 $\mathrm{MHz}): \delta 7.91\left(\mathrm{lH}, \mathrm{d}, J=1.96 \mathrm{~Hz}, \mathrm{H}-6^{\prime}\right) ; 7.51(\mathrm{lH}, \mathrm{dd} . J=1.96$ $\mathrm{Hz}$ and $\left.8.4 \mathrm{~Hz}, \mathrm{H}-5^{\prime}\right) ; 6.82\left(1 \mathrm{H}, \mathrm{d}, J=8.4 \mathrm{~Hz}, \mathrm{H}-2^{\prime}\right) ; 6.34$ $(\mathrm{lH}, \mathrm{d}, J=2.04 \mathrm{~Hz}, \mathrm{H}-8) ; 6.12(1 \mathrm{H}, \mathrm{d}, J=2.04 \mathrm{~Hz}, \mathrm{H}-6) ; 5.17$ (lH, d. J =7.76 Hz, H-1' '); (4.02-3.3.62, 6H, m, H-2' '-6" ' ; ${ }^{13} \mathrm{CNMR}$ (MeOD, $100 \mathrm{MHz}$ ): 177.05 (C-4), 164.91 (C-7), 164.27 (C-5), 161.82 (C-9), 159.30 (C-2), 157.58 (C-4'), $154.69\left(\mathrm{C}-3^{\prime}\right), 133.58$ (C-3), 123.07 (C-6'), 123.07 (C-2'), $117.49\left(\mathrm{C}-5^{\prime}\right), 116.61\left(\mathrm{C}-1^{\prime}\right), 106.35$ (C-10), 102.14 (C$\left.1^{\prime \prime}\right), 101.31$ (C-6), 98.10 (C-8), 78.32 (C-5' '), 77.54 (C$\left.3^{\prime \prime}\right), 73.52$ (C-2'”), 70.45 (C-4'”), 62.25 (C-6' ') (20).

Compound 6: Kaempferol-3-O-arabinoside: obtained as fine yellow needles. ${ }^{1} \mathrm{H}$ NMR (MeOD, $\left.400 \mathrm{MHz}\right): \delta$ $7.98\left(2 \mathrm{H}, \mathrm{d}, J=8.88 \mathrm{~Hz}, \mathrm{H}=2^{\prime}, 6^{\prime}\right) ; 6.81(2 \mathrm{H}, \mathrm{d}, J=8.88 \mathrm{~Hz}$, $\left.\mathrm{H}=3^{\prime}, 5^{\prime}\right)$; $6.32(1 \mathrm{H}, \mathrm{d}, J=2.08 \mathrm{~Hz}, \mathrm{H}=8) ; 6.12(1 \mathrm{H}, \mathrm{d}, J$ $=2.04 \mathrm{~Hz}, \mathrm{H}=6) ; 5.06\left(1 \mathrm{H}, \mathrm{d}, J=6.24 \mathrm{~Hz}, \mathrm{H}=1{ }^{\prime \prime}\right) ;{ }^{13} \mathrm{CNMR}$ (MeOD, 100 MHz): 181.09 (C-4), 163.12 (C-7), 163.07 (C5), 161.94 (C-4'), 159.56 (C-9), 158.35 (C-2), 133.43 (C3), $130.92\left(\mathrm{C}-6^{\prime}\right), 129.60\left(\mathrm{C}-2^{\prime}\right), 123.49\left(\mathrm{C}-1^{\prime}\right), 114.68$ (C5',3'), 102.90 (C-10), 102.13 (C-1' '), 98.49 (C-6), 93.99 (C-8), $74.99\left(\mathrm{C}-5^{\prime \prime}\right), 73.61\left(\mathrm{C}-3^{\prime \prime}\right), 67.50\left(\mathrm{C}-2^{\prime \prime}\right), 63.32$ $\left(\mathrm{C}-6^{\prime \prime}\right)(21)$.
Compound 7: Kaempferol-4'-O-methylether: obtained as fine yellow needles. ${ }^{1} \mathrm{H}$ NMR (MeOD, $\left.400 \mathrm{MHz}\right): \delta$ $8.11\left(2 \mathrm{H}, \mathrm{d}, J=8.85 \mathrm{~Hz}, \mathrm{H}=2^{\prime}, 6^{\prime}\right) ; 6.93(2 \mathrm{H}, \mathrm{d}, J=8.85$ $\left.\mathrm{Hz}, \mathrm{H}=3^{\prime}, 5^{\prime}\right) ; 6.39$ (1H, d, $\left.J=2 \mathrm{~Hz}, \mathrm{H}=8\right) ; 6.21(1 \mathrm{H}, \mathrm{d}, J$ $=2 \mathrm{~Hz}, \mathrm{H}=6) ; 3.98\left(3 \mathrm{H}, s, \mathrm{OCH}_{3}\right) ;{ }^{13} \mathrm{CNMR}(\mathrm{MeOD}, 100$ $\mathrm{MHz}$ ): 186.71 (C-4), 172.04 (C-7), 169.20 (C-5), 162.96 (C-4'), 158.58 (C-9), 142.86 (C-2), 133.52 (C-3), 130.92 $\left(\mathrm{C}-6^{\prime}, 2^{\prime}\right), 121.69\left(\mathrm{C}-1^{\prime}\right), 114.73\left(\mathrm{C}-5^{\prime}, 3^{\prime}\right), 103.69$ (C-10), 99.19 (C-6), 97.69 (C-8), $68.68\left(\mathrm{OCH}_{3}\right)(22)$.

Compound 8: Kaempferol-3-O-glucoside: obtained as fine yellow needles. ${ }^{1} \mathrm{H}$ NMR (MeOD, $\left.400 \mathrm{MHz}\right): \delta$ $8.10\left(2 \mathrm{H}, \mathrm{d}, J=8.9 \mathrm{~Hz}, \mathrm{H}=2^{\prime}, 6^{\prime}\right) ; 6.88(2 \mathrm{H}, \mathrm{d}, J=8.8 \mathrm{~Hz}$, $\left.\mathrm{H}=3^{\prime}, 5^{\prime}\right) ; 6.39(1 \mathrm{H}, \mathrm{d}, J=1.92 \mathrm{~Hz}, \mathrm{H}=8) ; 6.19(1 \mathrm{H}, \mathrm{d}, J=2$ $\mathrm{Hz}, \mathrm{H}=6) ; 5.12$ (1H, d, J=7.8 hz, $\mathrm{H}=1^{\prime \prime}$ ) ; 3.08-3.66 (m, rest of glucose protons); ${ }^{13} \mathrm{CNMR}(\mathrm{MeOD}, 100 \mathrm{MHz}): 178.01$ (C-4), 163.24 (C-7), 162.26 (C-5), 161.74 (C-4'), 155.86 (C-9), 156.55 (C-2), 133.53 (C-3), 130.92 (C-6',2'), 121.69 $\left(\mathrm{C}-1^{\prime}\right), 114.73$ (C-5',3'), 103.69 (C-10), 102.63 (C-1' '), 99.19 (C-6), 94.15 (C-8), 77.47 (C-5' '), 73.89 (C-3' '), $68.68\left(\mathrm{C}-2^{\prime \prime}\right), 62.58\left(\mathrm{C}-6^{\prime \prime}\right)$ (23) (Table 1 and Figure 1$)$.

HPLC-MS/MS metabolites profiling

HPLC-MS/MS proved in the last scientific decades its importance in recognition of phytochemical components of the plant extracts. The MS/MS spectrum is a significant 
fingerprint for every compound in the extract, despite the similarity of the molecular formula (35). In Table 3, we exhibited the recognition of seventy compounds from the hydroalcoholic extract of $H$. flavum. Phytochemical constituents were tentatively recognized by contrasting their molecular weights, retention time (Rt), and tandem mass (MS/MS) fragmentation model with formerly reported literature. Figure 2 shows the HPLC-ESI-MS/MS base peak chromatogram of the H. flavum extract. Seventy compounds, up till now, were recognized from the leaf extract of $H$. flavum organized into hydroxycinnamic acid derivatives, flavonoids, and other miscellaneous compounds.

Table 3. Compounds tentatively identified from $H$. flavum hydroalcoholic extract via HPLC-MS/MS

\begin{tabular}{|c|c|c|c|c|c|}
\hline Peak no. & Ret. & [M-H]- & Compound & Fragments & Ref. \\
\hline 1 & 0.50 & 206 & Benzyl succinic acid & $192,180,162,161$ & (36) \\
\hline 2 & 0.60 & 769 & Isorhamnetin-3-O-(2",3"-O-dirhamnosyl) glucoside & $747,723,702,665,638,497$ & (52) \\
\hline 3 & 0.86 & 317 & Myricetin & $299,225,189,165,149,125,95$ & (53) \\
\hline 4 & 1.01 & 461 & Kaempferol-glucuronide & $315,297,285,207,179,176,163,152$ & (37) \\
\hline 5 & 1.34 & 191 & Quinic acid & $173,155,127,109,93$ & (36) \\
\hline 6 & 1.54 & 609 & Kaempferol -O-diglucoside & $489,429,381,285,255,229$ & (50) \\
\hline 7 & 1.63 & 133 & Malic acid & $115,89,87,73,70,59$ & (36) \\
\hline 8 & 1.67 & 609 & $\begin{array}{l}\text { Quercetin 3-O- } \alpha \text {-L-rhamnopyranosyl-(1-6)- } \beta \text {-D- } \\
\text { glucopyranoside }\end{array}$ & 429,301 & (4) \\
\hline 9 & 2.15 & 353 & caffeoylquinic acid (Chlorogenic acid) & $309,237,191,179,173,161,135$ & (37) \\
\hline 10 & 2.47 & 337 & Coumaroylquinic acid & $247,203,173,163,191$ & (38) \\
\hline 11 & 2.59 & 739 & Kaempferol-rhamnoside-hexoside-rhamnoside & $695,610,587,557,497,374,336,285,235$ & $(42)$ \\
\hline 12 & 2.64 & 675 & Feruloyl-O-p-coumaroyl-O-caffeoylshikimic acid & $586,558,540,489,427,399,378,337,316,191$ & (39) \\
\hline 13 & 2.86 & 625 & $\begin{array}{l}\text { Quercetin 3-O-(2"-O- } \beta \text {-D-glucopyranosyl)- } \beta \text {-D- } \\
\text { galactopyranoside }\end{array}$ & $\begin{array}{l}607,583,505,463,445,409,355,325,301,255, \\
179\end{array}$ & $(48)$ \\
\hline 14 & 3.21 & 515 & dicaffeoylquinic acid & $370,353,299,255,240,179,155$ & (54) \\
\hline 15 & 3.25 & 609 & Isorhamnetin-O-hexoside-pentoside & $516,447,393,357,327,309,255$ & (55) \\
\hline 16 & 3.89 & 595 & Quercetin-3-O-glucosyl-pentoside & $415,301,271,255,229$ & (49) \\
\hline 17 & 3.98 & 677 & 3,4,5-tricaffeoylquinic acid & $515,497,469,411,335,272$ & (41) \\
\hline 18 & 4.02 & 933 & $\begin{array}{l}\text { Quercetin-3,40-di-glucoside-30-(6-p-coumaroyl- } \\
\text { glucoside) }\end{array}$ & $771,676,625,608,591,536,445,367,325,301$ & $(56)$ \\
\hline 19 & 4.19 & 593 & Kaempferol-rhamno-glucoside & $557,523,441,427,315,299,285,225$ & (57) \\
\hline 20 & 4.40 & 963 & Quercetin-3,40-di-glucoside-30-(6-feruloyl-glucoside) & $711,638,625,609,517,485,315$ & $(56)$ \\
\hline 21 & 4.45 & 917 & kaempferol-3-O-coumaroyldiglucoside-7-O-glucoside & $755,725,620,609,593,565,513,393,285$ & $(58)$ \\
\hline 22 & 4.63 & 947 & Kaempferol-O-trihexoside-hexuronoide & $785,623,609,592,535,429,358,285$ & (55) \\
\hline 23 & 4.76 & 579 & Kaempferol 3-O-hexoside pentoside & $417,399,381,309,285,255,187$ & (51) \\
\hline 24 & 5.00 & 771 & Quercetin 7-O-glucoside-3-O-rutinoside & $\begin{array}{l}727,625,609,595,539,505,427,325,301,273, \\
241\end{array}$ & $(59)$ \\
\hline 25 & 5.10 & 499 & 3-O-caffeoyl-4-O-p-coumaroylquinic Acid & $463,383,353,337,335,282,222,163$ & $(42)$ \\
\hline 26 & 5.20 & 771 & Quercetin-3-O-glucosyl-pentoside-7-O-glucuronide & $755,625,609,595,563,420,387,301,271$ & (49) \\
\hline 27 & 5.24 & 801 & dicaffeoyl-protocatechuic acid diglucoside & $747,681,639,625,607,577,325,271,243$ & (43) \\
\hline 28 & 5.76 & 755 & Quercetin-di-(rhamno)-hexoside & $717,609,591,489,447,429,367,285,257$ & $(57)$ \\
\hline 29 & 5.80 & 447 & Quercetin-3-O- $\alpha$-L-rhamnopyranoside & $432,393,357,301,255,192,179,151$ & (60) \\
\hline 30 & 6.22 & 477 & Isorhamnetin-3-O-glucoside & $449,357,315,299,285,189,151$ & (36) \\
\hline 31 & 6.46 & 785 & Kaempferol-O-dihexoside-hexuronoide & $623,609,591,485,429,339,285$ & (55) \\
\hline 32 & 6.51 & 756 & Quercetin 3-O-(2",6"'-di-O-rhamnosyl) Glucoside & $609,429,367,285$ & (61) \\
\hline 33 & 6.63 & 447 & Kaempferol-glucoside & $376,321,299,285,179,151$ & (37) \\
\hline 34 & 6.74 & 787 & Quercetin-tri-O-hexoside & $767,699,623,609,485,429,393$ & $(62)$ \\
\hline 35 & 6.88 & 499 & Coumaric acid derivative & $480,466,453,423,393,353,299,255,203,173$ & (36) \\
\hline 36 & 7.25 & 845 & Triacylglycerol & $\begin{array}{l}285,309,321,358,411,447,477,521,580,605 \\
609,682,698,755,809\end{array}$ & (36) \\
\hline 37 & 8.50 & 431 & Kaempferol-Rhamnoside & $387,357,299,285,243,181,151,137$ & (36) \\
\hline 38 & 9.03 & 193 & Caffeic acid methyl ester & $178,161,147,134$ & (45) \\
\hline 39 & 9.07 & 501 & Coumaric acid derivative & $377,337,323,307,280,263,179,163,149$ & (44) \\
\hline 40 & 9.43 & 979 & $\begin{array}{l}\text { Quercetin-3,4'-di-glucoside-3'-(6-methoxycaffeoyl } \\
\text { glucoside) }\end{array}$ & $\begin{array}{l}960,917,858,815,779,728,669,609,573,539 \\
476,447,413,301\end{array}$ & $(56)$ \\
\hline 41 & 10.23 & 755 & $\begin{array}{l}\text { Quercetin-3-O-alpha-L-rhamnopyranosyl(1-2)-beta-D- } \\
\text { glucopyranoside-7-O-alpha-L-rhamnopyranoside }\end{array}$ & $723,609,505,463,447,397,343,301,271$ & (36) \\
\hline
\end{tabular}


Table 3. Continued

\begin{tabular}{|c|c|c|c|c|c|}
\hline Peak no. & Ret. & [M-H]- & Compound & Fragments & Ref. \\
\hline 42 & 10.65 & 693 & $\begin{array}{l}\text { 5-O-p-coumaroyl-4-O-caffeoyl-4-methylpentanoic acid- } \\
\text { 5-hydroxy-3-quinate }\end{array}$ & $675,609,591,573,489,327,285,255$ & (39) \\
\hline 43 & 11.38 & 739 & Kaempferol-3-Glucoside-2"-Rhamnoside-7-Rhamnoside & $592,489,447,430,429,393,309,285,257,255$ & (36) \\
\hline 44 & 11.48 & 683 & Caffeic acid hexoside dimer & $\begin{array}{l}624,565,531,489,437,417,399,389,285,255, \\
227\end{array}$ & (44) \\
\hline 45 & 12.00 & 567 & Quercetin 3-O-(6"'-O-benzoyl) Galactoside & $\begin{array}{l}565,549,531,447,395,337,301,285,243,193, \\
175\end{array}$ & (61) \\
\hline 46 & 12.10 & 529 & caffeoyl-feruloylquinic acid & $452,439,393,367,349,311,193,179,161$ & (54) \\
\hline 47 & 12.46 & 993 & Quercetin-3,4'-di-glucoside-3'-(6-sinapoyl-glucoside) & $916,911,876,831,814,647,422,405,301$ & (56) \\
\hline 48 & 12.52 & 623 & Rhamnosylhexosyl methyl quercetin & $\begin{array}{l}579,477,461,429,383,323,299,285,263,221, \\
179\end{array}$ & (39) \\
\hline 49 & 12.57 & 577 & Kaempferol-3-O-rhamnoside-7-O-rhamnoside & $577,507,398,299,225$ & (36) \\
\hline 50 & 13.54 & 177 & Methoxycinnamic acid & $162,145,135,118$ & (36) \\
\hline 51 & 13.72 & 593 & Kaempferol rutinoside & $447,413,285$ & (63) \\
\hline 52 & 13.77 & 327 & Trihydroxy-octadecadienoic acid isomer & $309,291,239,229,221,211,209,197,171$ & (39) \\
\hline 53 & 14.07 & 709 & $\begin{array}{l}\text { Kaempferol } \\
\text { dirhamnosylpentoside }\end{array}$ & $561,399,285,255$ & (64) \\
\hline 54 & 14.60 & 769 & Isorhamnetin-3-O-(2",3"'-O-dirhamnosyl) galactoside & $\begin{array}{l}753,733,710,625,606,563,521,510,445,420, \\
359,335,254,221\end{array}$ & $(52)$ \\
\hline 55 & 14.91 & 579 & Quercetin-3-O- $\alpha$-L-arabinosyl-(1 $\rightarrow 2)$ - $\alpha$-L-rhamnoside & $302,299,225$ & $(65)$ \\
\hline 56 & 15.48 & 555 & Ferulic acid derivative & $299,243,225,207,183,165$ & (44) \\
\hline 57 & 16.27 & 329 & Quercetin dimethyl ether & $311,301,283,273,257,209,201,171,125,113$ & (51) \\
\hline 58 & 16.92 & 757 & Quercetin-O-dihexoside-pentoside & $728,688,609,430,393,351,301,285$ & (66) \\
\hline 59 & 18.90 & 309 & Eicosenoic acid & $291,273,251,183,171,153,135$ & (36) \\
\hline 60 & 19.66 & 285 & Kaempferol & 285 & (37) \\
\hline 61 & 20.06 & 827 & tricaffeoyl-glucosyl glucoside. & $736,685,615,601,595,551,491,353$ & (43) \\
\hline 62 & 21.06 & 691 & dicaffeoyl-feruloylquinic acids & 415, 397, 323, 293, 285 & (46) \\
\hline 63 & 21.21 & 958 & Hydroxyquercetin-3-O-hexose dimer & $835,788,765,599,572,479,357$ & (67) \\
\hline 64 & 21.91 & 505 & Quercetin-acetyl-glycoside & $441,418,346,310,301,277,245,227,189,153$ & (68) \\
\hline 65 & 22.18 & 988 & Quercetin-acetyl-rutinoside hexoside glucuronide & $952,730,595,353,301$ & (68) \\
\hline 66 & 24.85 & 529 & 3,5-di-O-caffeoyl quinic acid methylester & $511,407,293,253$ & (47) \\
\hline 67 & 27.89 & 465 & Dihydroquercetin glucoside & $436,419,380,377,312,310,285,283$ & (69) \\
\hline 68 & 29.56 & 447.5 & Kaempferol-galactoside & $408,365,331,285$ & (70) \\
\hline 69 & 31.38 & 549 & Quercetin 3-(6 (malonylhexoside & $\begin{array}{l}530,520,498,473,463,418,381,368,310,301 \text {, } \\
245\end{array}$ & (71) \\
\hline 70 & 38.24 & 625 & Quercetin-di-glucoside & $524,499,463,301$ & (72) \\
\hline
\end{tabular}

\section{Hydroxycinnamic acid derivatives}

Nineteen different hydroxycinnamic acid derivatives were tentatively identified in $H$. flavum extract. At retention time $1.34 \mathrm{~min}$, a molecular ion peak appeared at $\mathrm{m} / \mathrm{z}$ [M$\mathrm{H}]^{-}$191, along with its fragmentation pattern assigned for Quinic acid (36). Peak 9 was proposed for Chlorogenic acid, as its fragmentation pattern and its molecular ion peak m/z [M-H] 353 was following (37). A molecular ion peak spotted at $\mathrm{m} / \mathrm{z}[\mathrm{M}-\mathrm{H}]^{-} 337$, assigned for Coumaroylquinic acid, as its fragmentation pattern was reported by Sánchez-Rabaneda et al (38). As documented by Said et al (39), peak 12 was identified as Feruloyl-O-pcoumaroyl-O-caffeoylshikimic acid with its molecular ion peak at $\mathrm{m} / \mathrm{z}[\mathrm{M}-\mathrm{H}]^{-}$675. At retention time 3.21 minutes, a molecular ion peak at $\mathrm{m} / \mathrm{z}[\mathrm{M}-\mathrm{H}]^{-} 515$ was characteristic for dicaffeoylquinic acid (40). Another caffeoylquinic acid derivative appeared at $\mathrm{m} / \mathrm{z}[\mathrm{M}-\mathrm{H}]^{-} 677$, was assigned for 3,4,5-tricaffeoylquinic acid (41). Peak 25 at m/z [M-H] 499 , allocated to 3 -O-caffeoyl-4-O-p-coumaroylquinic acid supported with its fragmentation pattern (42).

As formerly reported by Benayad et al (43), the molecular ion spotted at $[\mathrm{M}-\mathrm{H}]^{-} 801$ was identified as dicaffeoyl-protocatechuic acid diglucoside. We identified two coumaric acid derivatives (peaks 35 and 39) after comparing their retention times and molecular ion peaks $\mathrm{m} / \mathrm{z}[\mathrm{M}-\mathrm{H}]^{-} 499,501$, respectively, with literature $(36,44)$. Moreover, the appearance of a molecular ion peak at $\mathrm{m} / \mathrm{z}$ [M-H] 193 characteristics for caffeic acid methyl ester (45). At retention time 10.65 minutes, a molecular ion peak at $\mathrm{m} / \mathrm{z}[\mathrm{M}-\mathrm{H}]^{-} 693$, showed the presence of 5-O-pcoumaroyl-4-O-caffeoyl-4-methylpentanoic acid-5hydroxy-3-quinate, evidenced by its daughter fragments as reported by Said et al (39). Caffeic acid hexoside dimer traced at $\mathrm{m} / \mathrm{z}[\mathrm{M}-\mathrm{H}]^{-} 683$ (44). According to Jaiswal et al (40), that assigned the molecular ion peak seen at $\mathrm{m} / \mathrm{z}$ [MH] 529 to caffeoyl-feruloylquinic acid.

At retention time 13.54 minutes, a molecular ion peak at $\mathrm{m} / \mathrm{z}[\mathrm{M}-\mathrm{H}]^{-} 177$ was identified as methoxycinnamic acid 


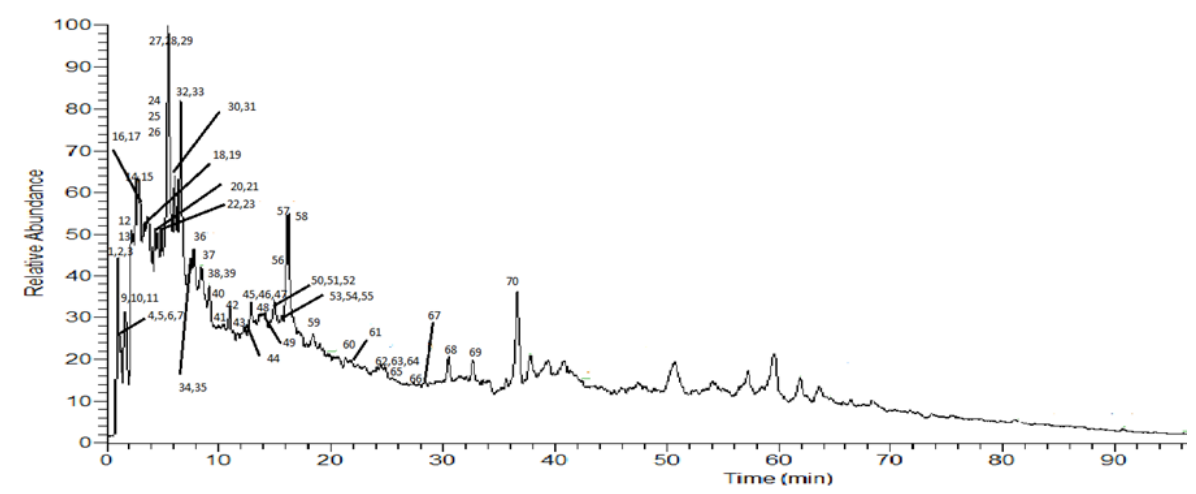

Figure 2. HPLC-ESI-MS/MS base peak chromatogram of the $H$. flavum extract.

(36). We can detect the ferulic acid derivative at $\mathrm{m} / \mathrm{z}$ [M$\mathrm{H}]^{-} 555$ (44), and a recognizable peak ion dedicated at $\mathrm{m} / \mathrm{z}$ [M-H] 827 for tricaffeoyl-glucosyl-glucoside, according to Benayad et al (43). Peaks $62 \& 66$ were recognized as two quinic acid derivatives according to their molecular ions and fragmentation models $(46,47)$.

\section{Flavonoids}

Forty-sixflavonoidsidentified in theLC/MSchromatogram of $H$. flavum, quercetin, and kaempferol were the main aglycones. Quercetin derivatives were previously isolated from the plant spotted in the chromatogram. At m/z [M$\mathrm{H}]^{-609}$, came out quercetin 3-O- $\alpha$-L-rhamnopyranosyl-(1 $\rightarrow 6)$ - $\beta$-D-glucopyranoside with the characteristic of quercetin ion at $\mathrm{m} / \mathrm{z}[\mathrm{M}-\mathrm{H}]^{-} 301$ (4). At retention time 2.86 minutes, a recognizable molecular ion peak appeared at $\mathrm{m} / \mathrm{z}[\mathrm{M}-\mathrm{H}]^{-} 625$, identified as quercetin $3-\mathrm{O}-\left(2^{\prime \prime}\right.$ O- $\beta$-D-glucopyranosyl)- $\beta$-D-galactopyranoside (48). It is noteworthy that we isolated this compound in this research. Another quercetin derivative reported herein as isolation and identification was spotted at retention time 3.89 minutes, with its molecular ion shown at $\mathrm{m} / \mathrm{z}$ [M$\mathrm{H}]^{-}$595, proposed for quercetin-3-O-glucosyl-pentoside (49). Moreover, the appearance of the deprotonated anion at $\mathrm{m} / \mathrm{z}[\mathrm{M}-\mathrm{H}]^{-} 447$, at retention time 5.80 minutes, was characteristic for quercetin-3-O- $\alpha$-L-rhamnopyranoside, previously isolated from the plant by Owis (4). On the other hand, kaempferol derivatives, formerly isolated from the plant, have been spotted in the spectrum at several molecular ion peaks. The isolation of kaempferol di-glucoside has been previously reported, where its deprotonated ion traced in our spectrum at $\mathrm{m} / \mathrm{z}[\mathrm{M}-\mathrm{H}]^{-}$ 609 (50). At retention time 4.76 minutes, a molecular ion peak at m/z [M-H] 579 was assigned for kaempferolhexoside-pentoside (51), where a similar compound was isolated and identified in this research as kaempferolglucoside-riboside. Kaempferol-glucoside was separated from the plant extract in our study, and recognized in the spectrum at retention time 6.63 , with its molecular ion at $\mathrm{m} / \mathrm{z}[\mathrm{M}-\mathrm{H}]^{-} 447$, and fragmentation pattern typical to that reported by Justesen (37).

\section{Docking studies}

We performed molecular docking of compounds (18) into the binding pockets of both RAF-1 and ERK-2 including the co-crystallized inhibitor (9) in each case. They got stabilized inside the binding pockets of RAF-1 and ERK-2 by promising scores and bound interactions with the amino acids of both receptors.

By analyzing the binding pockets of RAF-1 and ERK2 proteins containing the co-crystallized inhibitors, we found that: the co-crystallized inhibitor of RAF-1 was stabilized by forming five H-bonds with Cys424, Lys375, Ser428, and Ile355 amino acids. On the other hand, the co-crystallized inhibitor of ERK-2 was able to compose a covalent bond with Cys164, two H-bonds with Met106 and Ser 151, and a pi-H bond with Ile29.

We depicted the scores and the detailed binding modes of the isolated flavonoids (1-8) with the amino acids of both RAF-1 and ERK-2 pockets in Table 4 and Table 5 .

\section{In vitro results}

\section{Cytotoxicity assay}

We detected the cytotoxic concentration of 50\% $\left(\mathrm{CC}_{50}\right)$ of the indicated purified $H$. flavum extract on HepG2 cells and the normal hepatocytes using MTT and LDH production assay kit. Accordingly, the cells were peculated in 96-well/plate in a density of 10000 cells/well and were left overnight. Then, the cells were treated all night with more than one concentration of H. flavum agent (0-25 $\mathrm{mg} / \mathrm{mL}$ ). Interestingly, the cell viability rate of HepG2 cells was being interrupted at a low concentration of $H$. flavum treatment $(200 \mathrm{ug} / \mathrm{mL})$ and revealed $50 \%$ inhibition at the concentration of $600 \mathrm{ug} / \mathrm{ml}$. Meanwhile, the cell viability rate of the normal hepatocytes showed an undetectable toxic effect at the same concentrations of $H$. flavum treatment (Figures $3 \mathrm{~A}$ and $3 \mathrm{~B}$ ). The $\mathrm{CC}_{50}$ of $\mathrm{H}$. flavum agent on the normal cells was almost $1 \mathrm{mg} / \mathrm{mL}$ indicating that the plant agent might disturb the cancer cells at a low concentration without any detectable cytotoxic effect on the normal cells. The treated cells were checked for their $\mathrm{LDH}$ production, which is considered an indicator of the chemical-mediated cytotoxicity in HepG2 cells 
Table 4. The binding scores and modes of the examined isolated flavonoids from Hymenosporum flavum leaf extract (1-8) against both raf-1 and ERK-2 binding pockets compared to the docked co-crystallized inhibitor in each docking process (docked 9)

\begin{tabular}{|c|c|c|c|c|c|c|}
\hline No. & Compound & $\mathbf{R}^{\mathbf{a}}$ & $S^{b}$ & RMSD $^{\mathrm{c}}$ & Amino acid interactions & Distance (A) \\
\hline \multirow{6}{*}{1} & \multirow{6}{*}{$\begin{array}{l}\text { Quercetin-3-O-glucopyranosyl }(1 \rightarrow 2) \\
\text { ribopyranoside }\end{array}$} & \multirow{3}{*}{ raf-1 } & \multirow{3}{*}{-8.43} & \multirow{3}{*}{1.28} & Cys424/H-acceptor & 2.83 \\
\hline & & & & & Ser428/H-donor & 2.83 \\
\hline & & & & & Ser428/H-acceptor & 3.01 \\
\hline & & \multirow{3}{*}{ ERK-2 } & \multirow{3}{*}{-7.34} & \multirow{3}{*}{2.16} & Gln103/H-donor & 2.87 \\
\hline & & & & & Lys112/H-acceptor & 2.97 \\
\hline & & & & & Cys164/H-donor & 3.60 \\
\hline \multirow{5}{*}{2} & \multirow{5}{*}{$\begin{array}{l}\text { Kaempferol-3-O-glucopyranosyl } \\
(1 \rightarrow 2) \text { ribopyranoside }\end{array}$} & \multirow{3}{*}{ raf-1 } & \multirow{3}{*}{-8.15} & \multirow{3}{*}{1.97} & Ser357/H-donor & 2.98 \\
\hline & & & & & Asp486/H-donor & 3.01 \\
\hline & & & & & Ile355/H-donor & 3.15 \\
\hline & & \multirow{2}{*}{ ERK-2 } & \multirow{2}{*}{-7.31} & \multirow{2}{*}{1.77} & Lys112/H-acceptor & 2.79 \\
\hline & & & & & Ser151/H-donor & 2.84 \\
\hline & & & & & Asp486/H-donor & 2.77 \\
\hline & & raf-1 & -885 & 146 & Lys375/H-acceptor & 2.92 \\
\hline & & rat-1 & -8.85 & 1.40 & Cys424/H-acceptor & 3.06 \\
\hline 3 & Quercetin 3-O-(2"-0-D- & & & & Phe475/pi-H & 3.53 \\
\hline 3 & glucopyranosyl) D-galactopyranoside & & & & Lys112/H-acceptor & 2.98 \\
\hline & & & & & Met106/H-donor & 3.14 \\
\hline & & ERK-L & $-8.1 /$ & $1 . / 2$ & Ile29/H-donor & 3.20 \\
\hline & & & & & Met106/H-acceptor & 3.38 \\
\hline & & raf-1 & -8.18 & 1.41 & Cys424/H-donor & 3.21 \\
\hline 4 & Quercetin 3-glucoside (Isoquercitrin) & & & & Ser151/H-donor & 2.81 \\
\hline & uuercentin s-giucostae (Isoquercturn) & ERK-2 & -6.67 & 1.56 & Gln103/H-donor & 2.83 \\
\hline & & & & & Lys112/H-acceptor & 2.87 \\
\hline & & raf-1 & -7.54 & 1.43 & Cys424/H-donor & 2.81 \\
\hline 5 & Ouercetin 3-galactoside & & & & Lys112/H-acceptor & 2.93 \\
\hline J & Guetcetiri s-galactusiue & ERK-2 & -7.08 & 2.10 & Gln103/H-donor & 3.19 \\
\hline & & & & & Ile29/pi-H & 4.37 \\
\hline & & raf-1 & $-7,23$ & 1.96 & Cys424/H-donor & 3.01 \\
\hline 6 & Kaemprerol 3-U- $\alpha-\mathrm{L}-$ & & & & Cys424/H-acceptor & 3.04 \\
\hline & & ERK-2 & -6.55 & 1.08 & Lys112/H-acceptor & 3.11 \\
\hline & & & & & Cys424/H-donor & 2.89 \\
\hline & & raf-1 & -681 & 199 & Lys375/H-acceptor & 2.97 \\
\hline & Kaempferol 4'-methyl ether & rat-1 & -6.81 & 1.99 & Cys424/H-acceptor & 2.98 \\
\hline 7 & (Kaempferide) & & & & Phe475/pi-pi & 3.84 \\
\hline & & EPK 2 & 5,15 & 118 & Met106/H-donor & 2.85 \\
\hline & & ERK-L & -5.45 & 1.48 & Lys112/H-acceptor & 2.89 \\
\hline & & & & & Ser428/H-donor & 2.95 \\
\hline & & $\mathrm{rff}_{1}$ & 792 & 132 & Cys424/H-acceptor & 3.06 \\
\hline & & $r d t-1$ & -1.92 & 1.50 & Cys424/H-donor & 3.24 \\
\hline 8 & Kaemnferol 3-glucoside & & & & Lys375/pi-H & 4.34 \\
\hline 0 & Ademplerol s-giucoside & & & & Met106/H-donor & 2.79 \\
\hline & & ERK-2 & -683 & 218 & Lys112/H-acceptor & 2.93 \\
\hline & & & & & Asp165/H-donor & 2.94 \\
\hline & & & & & Met106/H-donor & 3.14 \\
\hline & & & & & Lys431/H-donor & 3.42 \\
\hline & & $m f_{1}$ & 715 & 175 & Cys424/H-acceptor & 3.48 \\
\hline & Co-crystallized inhibitor & ral-1 & -1.15 & $1 . / 2$ & Ile355/H-donor & 3.50 \\
\hline 9 & (Docked) & & & & Phe475/pi-H & 3.25 \\
\hline & & EPK & 605 & 20 & Ser151/H-donor & 2.70 \\
\hline & & ERK-2 & -6.05 & 2.08 & Ile29/pi-H & 4.24 \\
\hline & & & & & Lys375/H-acceptor & 2.75 \\
\hline & & raf-1 & & & Lys431/H-donor & 2.80 \\
\hline & & rat-1 & & & Ile355/H-donor & 3.31 \\
\hline 10 & Co-crystallized inhibitor & & & & Cys424/H-donor & 3.36 \\
\hline 10 & (Native) & & & & Cys164/Covalent & - \\
\hline & & & & & Ser151/H-donor & 2.69 \\
\hline & & ERK-L & & & Met106/H-donor & 3.00 \\
\hline & & & & & Ile29/pi-H & 4.45 \\
\hline
\end{tabular}

${ }^{a} \mathbf{R}$ : the target receptor pocket. ${ }^{\mathrm{b}} \mathbf{S}$ : the score of a compound inside the protein binding pocket (kcal/mol), ${ }^{\mathrm{c}} \mathbf{R M S D}$ : The root mean squared deviation between the predicted pose and the crystal structure. 
Table 5. $3 \mathrm{D}$ pictures of the binding interactions and the protein pockets positioning of the most promising isolated flavonoids from Hymenosporum flavum leaf extract

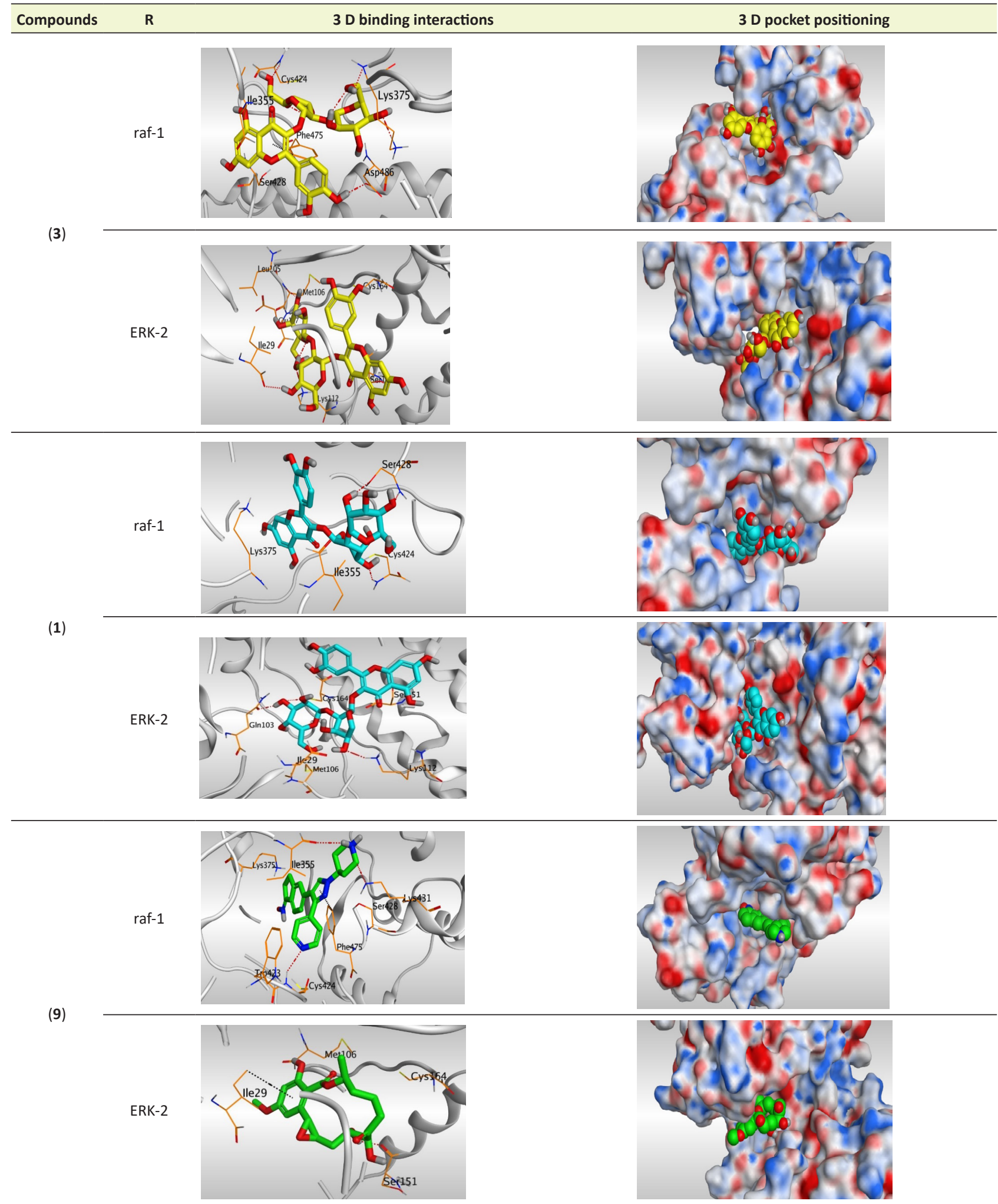

Compounds (1) and (3) against both raf-1 and ERK-2 receptors compared to the docked co-crystallized inhibitor (9) in each case, respectively. Red dash represents $\mathrm{H}$-bonds and black dash represents $\mathrm{H}$-pi interactions. 
A

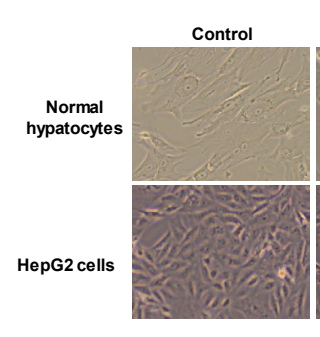

B
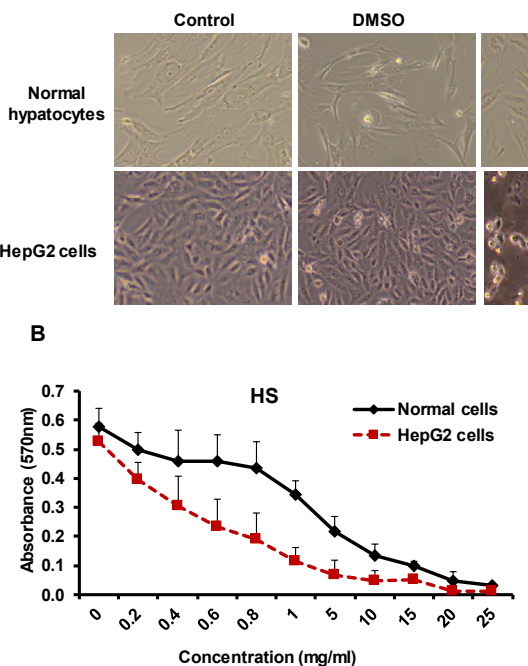

HS

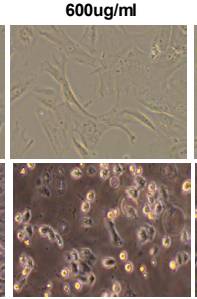

HS
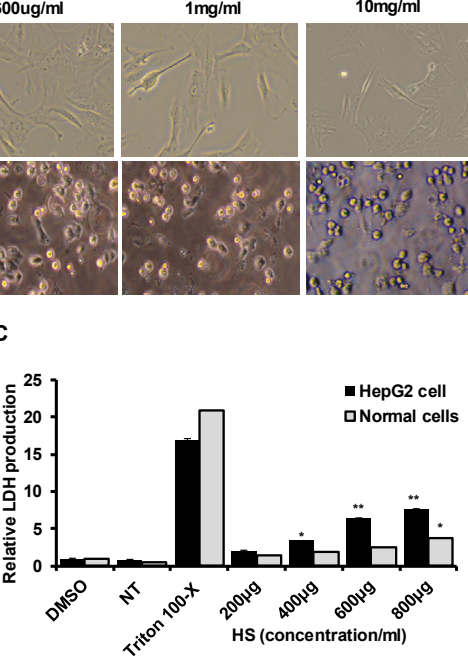

Figure 3. (A) Representative cell images revealing the cell viability of HepG2 and normal hepatocytes cell line pre-treated with Hymenosporum flavum extract in comparison with DMSO-treated cells and non-treated cells (control). (B) Calculated $\mathrm{CC}_{50}$-dependent cell viability rate of Hymenosporum flavum extract on normal hepatocytes cells, and HepG2 cells pre-treated with different concentrations (0-25 mg/mL) of Hymenosporum flavum extract using MTT assay. (C) Relative lactate dehydrogenase (LDH) production from treated and untreated cell (NT) compared with Triton 100-X treated cells.

Error bars indicate standard deviation (SD) of four different replicates. Student two-tailed test was used to determine P values and significance of LDH production level.

and normal cells. There was a significant elevation in the relative $\mathrm{LDH}$ production up to threefold in HepG2 cells treated with $200 \mathrm{ug} / \mathrm{ml}$ and gradually increased in a dosedependent manner compared to the normal hepatocytes (Figure 3C). This result further confirms the cytotoxic effect of $H$. flavum agent on HepG2 cells indicated by the production level of LDH upon treatment compared to the normal cells.

Reverse transcription and quantitative real time-PCR Table 6 shows the degree of gene expression of RAF-1 and Erk-2 genes in the HepG2 cell line after treatment with sorafenib, compound $\mathbf{1}$, compound 3 , and their combination. The gene expression levels of the RAF-1 and Erk-2 genes were tested in cell lines prior to the treatment with the $100 \mu \mathrm{g} / \mathrm{mL}$ of sorafenib, compound $\mathbf{1}$, compound $\mathbf{3}$, and their combination, along with a comparison to the

Table 6. Relative Raf-1 and Erk-2 gene expression in the HepG2 cell line according to sample data

\begin{tabular}{llcc}
\hline \multirow{2}{*}{ Ser. } & \multirow{2}{*}{ Sample } & \multicolumn{2}{c}{ Gene expression } \\
\cline { 3 - 4 } & & \multicolumn{2}{c}{ Fold Change } \\
\cline { 3 - 4 } & Code & \multicolumn{2}{c}{ fld } \\
\cline { 3 - 4 } & & 0.51173 & 0.59307 \\
2 & s5/HepG2 & 0.28716 & 0.41522 \\
3 & s7/HepG2 & 0.22457 & 0.27847 \\
4 & Sorafenib/HepG2 & 0.33076 & 0.39288 \\
5 & s5+ Sorafenib/HepG2 & 0.18561 & 0.24325 \\
6 & Control HepG2 & 1 & 1 \\
\hline
\end{tabular}

control using PCR.

Figure 4 shows the gene expression patterns for (a) RAF1 and (b) ERK-2 in hepatocellular carcinoma (HepG2) cell lines, estimated by real-time PCR. The order of gene expression inhibition for the three estimated samples was as follows: sorafenib $>$ compound $\mathbf{1}>$ compound $\mathbf{3}$. The inhibitory effect of sorafenib with compound $\mathbf{1}$ on both RAF-1 and ERK-2 genes was superior to sorafenib alone, which illuminates the strong inhibition of this combination on the RAF/MEK/ERK pathway. The results disclose the underlying molecular mechanisms formerly stated results of the cytotoxic assay. Our results revealed that compound 1 enhanced the cytotoxic effect of sorafenib on HepG2 and decreased cell viability (downregulated the gene expression of both RAF-1 and Erk-2).

\section{Discussion}

Two new flavonoids (compounds $\mathbf{1}$ and 2) were isolated from Hymenosporum flavum together with the other six known compounds. Compound $\mathbf{1}$ was obtained as a yellow powder, with molecular formula $\mathrm{C}_{26} \mathrm{H}_{28} \mathrm{O}_{16}$, assigned by ESI-MS, showing a molecular ion peak at $\mathrm{m} / \mathrm{z} 595.3771$ $[\mathrm{M}-\mathrm{H}]^{-}$in HR-ESI-MS, along with daughter fragments at $\mathrm{m} / \mathrm{z}[\mathrm{M}-\mathrm{H}]^{-} 433.0857$, due to loss of glucoside moiety, and $\mathrm{m} / \mathrm{z}[\mathrm{M}-\mathrm{H}]^{-} 301.1429$ due to loss of riboside moiety (Figure 1 and Table 2).

The aromatic protons at $\delta \mathrm{H} 6.23(\mathrm{~d}, J=2 \mathrm{~Hz}, \mathrm{H}-6)$, 6.41 (d, J=2 Hz, H-8), 6.94 (d, J=8.4 Hz, H-5'), 7.63 (d, $\left.J=2.04 \mathrm{~Hz}, \mathrm{H}-2^{\prime}\right)$, and $7.62\left(\mathrm{dd}, J=8.4 \mathrm{~Hz}, 2.04 \mathrm{~Hz}, \mathrm{H}-6^{\prime}\right.$ ) designated a quercetin flavonol. Two anomeric proton doublets were confirmative for two sugar moieties. The 

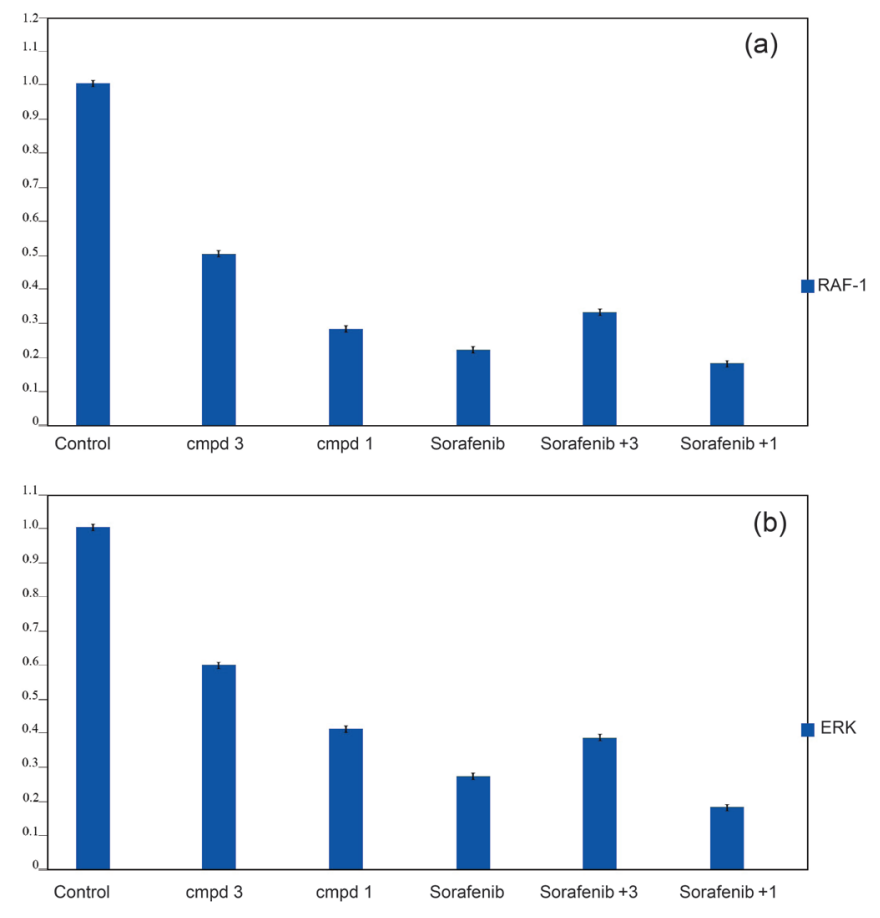

Figure 4. Gene expression patterns for Raf-1 (a) and Erk-2 (b). Cmpd: compound.

first signal at $\delta \mathrm{H} 5.41\left(\mathrm{H}-1^{\prime \prime}\right)$ with its coupling constant $(J=3.8 \mathrm{~Hz})$, which was less than $4.0 \mathrm{~Hz}$, revealed the a-configuration of the ribose moiety, excluding arabinose and xylose moieties (33). The second one at $\delta \mathrm{H} 4.57$ along with its coupling constant $\left(J=7.76 \mathrm{~Hz}, \mathrm{H}-1^{\prime \prime \prime}\right)$ illustrated the beta configuration of the glucose residue.

The ${ }^{13} \mathrm{CNMR}$ signals (Table 2) affirmed the presence of 15 flavonol carbon signals, confirming the quercetin aglycone after comparing the NMR data with literature (33). The remaining carbon signals (Table 2) proved the existence of two sugar moieties, which deducted ribose and glucose after comparing the NMR data with literature $(33,34)$. A downfield shift of C-2" ( $\delta 78.3$, ribose) also supported the attachment of the glucopyranosyl unit.

Further confirmation was carried out by ${ }^{13} \mathrm{CNMR}$ where the shift of C-3 up-field (from $\delta$ C 135.8 to 134.3,), while that of C-2 downfield (from $\delta$ C 146.9 to 157.07) when compared with quercetin (33). Consequently, compound 1 was elucidated as quercetin-3-(glucopyranosyl $1 \rightarrow 2$ ribopyranoside), where this is the first time isolated in nature.

Compound 2 isolated as yellow needles displayed a dark purple spot on paper chromatogram under UV light, which became yellow upon exposure to ammonia vapor. It has a molecular formula $\mathrm{C}_{26} \mathrm{H}_{29} \mathrm{O}_{15}$, which is deducted by HR-ESI-MS m/z 579.3777 [M-H]', and daughter fragments $\mathrm{m} / \mathrm{z}[\mathrm{M}-\mathrm{H}]^{-} 417.0659$, and $\mathrm{m} / \mathrm{z}$ [M$\mathrm{H}]^{-}$285.1780, respectively, due to glucoside and riboside moieties loss (Table 2).

In the ${ }^{1} \mathrm{H}$ NMR spectrum, signals appeared at $\delta \mathrm{H}: 6.11$ (d, J=2.2 Hz, H-6), 6.31 (d, J=2.2 Hz, H-8), 6.83 (d, J=8.76 $\left.\mathrm{Hz}, 2 \mathrm{H}, \mathrm{H}-3^{\prime}, \mathrm{H}-5^{\prime}\right)$, and 7.92 (d, J=8.76 Hz, 2H, H-2',
H-6') designated a kaempferol flavonol. Two anomeric proton doublets were confirmative for two sugar moieties. The first signal at $\delta \mathrm{H} 5.38$ ( $\left.\mathrm{H}-1^{\prime \prime}\right)$ besides its coupling constant $(J=3.36 \mathrm{~Hz})$ lower than $4.0 \mathrm{~Hz}$, revealed ribose moiety $\alpha$-configuration, after the exclusion of arabinose and xylose sugars (33). The other signal at $\delta \mathrm{H} 4.46$ with its coupling constant $\left(J=7.72 \mathrm{~Hz}, \mathrm{H}-1^{\prime \prime \prime}\right)$ evidenced the beta configuration of the glucose sugar.

The ${ }^{13} \mathrm{C}$ NMR signals (Table 2) affirmed the 15 carbon signals of a flavonol, as compared with literature, confirmed the kaempferol aglycone (33). Other ${ }^{13} \mathrm{C}$ NMR signals showed two sugar moieties assigned for ribose and glucose after comparison with literature $(23,33)$. A downfield shift of C-2" ( $\delta 78.6$, ribose) also evidenced the attachment of glucopyranosyl unit. Further confirmation was carried out by C-3 up-field shift (from $\delta$ C 135.5 to 132.7) and C-2 downfield shift (from $\delta$ C 146.8 to 155.16), in comparison with kaempferol aglycone (33). Hence, compound 2 was recognized as kaempferol-3(glucopyranosyl $1 \rightarrow 2$ ribopyranoside), where is the first time isolated in nature.

The docking simulation results revealed that all the isolated flavonoids (1-8), especially compounds (1) and (3) achieved the best binding scores towards both RAF1 and ERK-2 receptors, which exceed the bound scores of the docked co-crystallized inhibitors (9) in each case (Table 4). However, compound (3) binding energy on RAF-1 and ERK-2 pockets were -8.85 and $-8.17 \mathrm{kcal} / \mathrm{mol}$ compared to -7.15 and $-6.05 \mathrm{kcal} / \mathrm{mol}$ of the docked cocrystallized inhibitor (9) in each case, respectively. The binding scores of compound (1) towards RAF-1 and ERK2 binding sites were -8.43 and $-7.34 \mathrm{kcal} / \mathrm{mol}$ compared 
to -7.15 and $-6.05 \mathrm{kcal} / \mathrm{mol}$ of the docked co-crystallized inhibitor (9) in each case, respectively.

Furthermore, the detailed binding modes of compounds (1) and (3) inside both RAF-1 and ERK-2 receptor pockets were similar to the native co-crystallized inhibitor (10) in binding with nearly the similar crucial amino acids superior to the docked co-crystallized one (9) in each case, respectively (Table 5).

We tried to clarify the role of quercetin glycosides as safe and efficacious anticancer agents. Interestingly, the cell viability rate of HepG2 cells was interrupted at a low concentration of the plant treatment. Meanwhile, the cell viability rate of the normal hepatocytes showed an undetectable toxic effect at the same concentrations of the plant treatment. The relative $\mathrm{LDH}$ production was significantly elevated threefold in HepG2 cells treated with $200 \mathrm{ug} / \mathrm{ml}$. It gradually increased in a dose-dependent manner when compared to normal hepatocytes. These findings indicate that $H$. flavum treatment is safe at both low and high doses in normal cells, where the extract provides a potent anticancer treatment.

According to the obtained docking results of the eight isolated flavonoids (1-8) of $\mathrm{H}$. flavum extract compared to the co-crystallized inhibitors of both RAF-1 and ERK2 receptor pockets, expressed promising idea about their affinities towards these carcinogenic proteins and subsequently expected high efficacies and intrinsic activities in their inhibition as well. As well, this study expected to be hopeful of the isolated flavonoids (1-8) of $H$. flavum leaf extract against raf-1 and ERK-2 receptors, especially compounds (1) and (3) either alone or in combinations with sorafenib for cancer treatment.

The current study concluded a synergistic combination of sorafenib with $H$. flavum and a combination of sorafenib with naturally purified quercetin glycosides that sensitize HCC cells towards sorafenib-induced apoptosis. We studied apoptosis-related genes' expression to unravel the underlying molecular mechanisms of the synergistic antitumor effects of $H$. flavum compounds and sorafenib on hepatocellular carcinoma cell line (HepG2). Therefore, we explored the biological activities of all compounds at a lower dose $(100 \mu \mathrm{g} / \mathrm{mL})$.

Many studies have shown that sorafenib depends on inducing tumor cell apoptosis in multi-cancers. Its mechanism depends on inhibiting the RAF/MEK/ ERK signaling pathway through suppressing tumor cell proliferation and the restriction process of angiogenesis (73). The mechanisms actions of sorafenib are diminishing cell growth, inducing cell cycle arrest in the G0/G1 phase, up regulation of the proapoptotic proteins (caspase 8 and caspase 3), and downregulation of the cell cycle-associated protein cyclin D1 and the anti-apoptotic protein MCL1. Sorafenib decreased the phosphorylation of ERK and MEK (74). Two pathways inhibited ERK in direct and indirect ways. It is better to directly prevent the
ERK protein pathway due to its multiple cellular functions as well as organizing the distribution of upstream signals to its nuclear and cytosolic effectors. We succeeded in the extraction of two natural compounds that could inhibit the ERK pathway directly, so that compounds 1 \& 3 inhibited ERK pathway alone. Also, both of them improved the efficiency of sorafenib in targeting the ERK pathways. Compounds 1 \& 3 also inhibited the ERK pathways indirectly by targeting Raf- 1 . Raf- 1 can activate RAS proteins and indicated that phosphorylation of serine 43 in Raf- 1 is responsible for disruption of Ras/Raf association and downstream signaling $(8,75)$. Interestingly, compounds $1 \& 3$ contributed to overcoming the resistance of HCC. Our findings showed a synergistically inhibitory effect on both Erk-1 and Raf-1 proteins interaction by compounds $\mathbf{1}$ and $\mathbf{3}$ in cancer cells.

The safety and inhibitory effects of sorafenib/compound 1 combined with cancer cell indicated that the use of compound 1 should be investigated in the future as a potential complementary to support the application of sorafenib therapy in HCC.

\section{Conclusion}

Two newly identified flavonols; quercetin-3-O(glucopyranosyl $1 \rightarrow 2$ ribopyranoside) (1) and kaempferol3-O-(glucopyranosyl $1 \rightarrow 2$ ribopyranoside) (2), along with other six flavonoids, were isolated from the leaf extract of $H$. flavum. Moreover, we identified seventy compounds from the HPLC-PDA/MS/MS of the hydroalcoholic extract, for the first time. The cytotoxic activity of the plant extract confirmed its potential action on HepG2 cells indicated by the production level of LDH upon treatment compared with the normal cells. Furthermore, compounds 1 and 3, which showed the best results in silico were further examined in vitro using qRT-PCR. They exhibited promising inhibitory activities against both RAF-1 and ERK-2 gene expressions. Also, both of them improved the efficiency of sorafenib in targeting both RAF-1 and ERK-2 pathways indicating synergistic combinations confirmed by the in vitro results of the MTT assay and PCR. The results revealed that compounds $\mathbf{1}$ and $\mathbf{3}$ could regulate the division of the tumor cells by restoring the sustained RAS/RAF/ERK signaling pathway and managing the programmed cell death. This mechanism showed that the isolated glycosides are safe in cancer cells' treatment with a noticeable effect on cell proliferation and angiogenesis. Our findings could be promising for further preclinical and clinical studies on the studied compounds, especially for compounds $\mathbf{1}$ and 3, either alone or in combinations with sorafenib for cancer treatment.

\section{Authors' contribution}

RFT and WAE designed the study, conducted the phytochemical analysis, and wrote the manuscript. EAK supervised the work, and critical revision of the article. 
On the other hand, AIA, HK and AH implemented the animal experiment, biochemical and gene expression analysis. AAA conducted the molecular docking studies and contributed to the manuscript writing. All authors read and approved the final version and agreed to publish it.

\section{Conflict of interests}

The authors declared no competing interests.

\section{Ethical considerations}

Not applicable.

\section{Funding/Support}

This research did not receive any specific grant from funding agencies in the public, commercial, or not-forprofit sectors.

\section{References}

1. Veeresham C. Natural products derived from plants as a source of drugs. J Adv Pharm Technol Res. 2012;3(4):200-1. doi: 10.4103/2231-4040.104709.

2. Al-Rimawi F, Rishmawi S, Ariqat SH, Khalid MF, Warad I, Salah Z. Anticancer activity, antioxidant activity, and phenolic and flavonoids content of wild Tragopogon porrifolius plant extracts. Evid Based Complement Alternat Med. 2016;2016:9612490. doi: 10.1155/2016/9612490.

3. Harrison L. RHS Latin for Gardeners: Over 3000 Plant Names Explained and Explored. Mitchell Beazley; 2012.

4. Owis AI. Phytochemical investigation of ethyl acetate extract of Hymenosporum flavum (Pittosporaceae) and its antimicrobial activity. Int J Appl Res Nat Prod. 2016;9(3):315 .

5. Motola-Kuba D, Zamora-Valdés D, Uribe M, MéndezSánchez N. Hepatocellular carcinoma. An overview. Ann Hepatol. 2006;5(1):16-24.

6. Huynh H, Nguyen TT, Chow KH, Tan PH, Soo KC, Tran E. Over-expression of the mitogen-activated protein kinase (MAPK) kinase (MEK)-MAPK in hepatocellular carcinoma: its role in tumor progression and apoptosis. BMC Gastroenterol. 2003;3:19. doi: 10.1186/1471-230x-3-19.

7. Wilhelm S, Carter C, Lynch M, Lowinger T, Dumas J, Smith RA, et al. Discovery and development of sorafenib: a multikinase inhibitor for treating cancer. Nat Rev Drug Discov. 2006;5(10):835-44. doi: 10.1038/nrd2130.

8. Häfner S, Adler HS, Mischak H, Janosch P, Heidecker G, Wolfman A, et al. Mechanism of inhibition of Raf- 1 by protein kinase A. Mol Cell Biol. 1994;14(10):6696-703. doi: 10.1128/mcb.14.10.6696-6703.1994.

9. Wellbrock C, Karasarides M, Marais R. The RAF proteins take centre stage. Nat Rev Mol Cell Biol. 2004;5(11):875-85. doi: $10.1038 / \mathrm{nrm} 1498$.

10. Seger R, Krebs EG. The MAPK signaling cascade. FASEB J. 1995;9(9):726-35.

11. Omar HA, Tolba MF, Hung JH, Al-Tel TH. OSU-2S/ Sorafenib synergistic antitumor combination against hepatocellular carcinoma: the role of $\mathrm{PKC} / \mathrm{p} 53$. Front Pharmacol. 2016;7:463. doi: 10.3389/fphar.2016.00463.

12. Kim C, Baek SH, Um JY, Shim BS, Ahn KS. Resveratrol attenuates constitutive STAT3 and STAT5 activation through induction of PTPE and SHP-2 tyrosine phosphatases and potentiates sorafenib-induced apoptosis in renal cell carcinoma. BMC Nephrol. 2016;17:19. doi: 10.1186/s12882016-0233-7.

13. Ku CY, Wang YR, Lin HY, Lu SC, Lin JY. Corosolic acid inhibits hepatocellular carcinoma cell migration by targeting the VEGFR2/Src/FAK pathway. PLoS One. 2015;10(5):e0126725. doi: 10.1371/journal.pone.0126725.

14. Rong LW, Wang RX, Zheng XL, Feng XQ, Zhang L, Zhang $\mathrm{L}$, et al. Combination of wogonin and sorafenib effectively kills human hepatocellular carcinoma cells through apoptosis potentiation and autophagy inhibition. Oncol Lett. 2017;13(6):5028-34. doi: 10.3892/ol.2017.6059.

15. Brogi S. Computational approaches for drug discovery. Molecules. 2019;24(17):3061. doi: 10.3390/ molecules24173061.

16. Abd El Maksoud AI, Taher RF, Gaara AH, Abdelrazik E, Keshk OS, Elawdan KA, et al. Selective regulation of B-Raf dependent K-Ras/mitogen-activated protein by natural occurring multi-kinase inhibitors in cancer cells. Front Oncol. 2019;9:1220. doi: 10.3389/fonc.2019.01220.

17. Ghanem A, Emara HA, Muawia S, Abd El Maksoud AI, AlKarmalawy AA, Elshal MF. Tanshinone IIA synergistically enhances the antitumor activity of doxorubicin by interfering with the PI3K/AKT/mTOR pathway and inhibition of topoisomerase II: in vitro and molecular docking studies. New J Chem. 2020;44(40):17374-81. doi: 10.1039/ D0NJ04088F.

18. Sobeh M, Mahmoud MF, Abdelfattah MAO, Cheng H, ElShazly AM, Wink M. A proanthocyanidin-rich extract from Cassia abbreviata exhibits antioxidant and hepatoprotective activities in vivo. J Ethnopharmacol. 2018;213:38-47. doi: 10.1016/j.jep.2017.11.007.

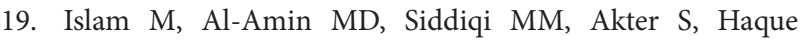
MM, Sultana N, et al. Isolation of quercetin-3-O-beta-Dglucopyranoside from the leaves of Azadirachta indica and antimicrobial and cytotoxic screening of the crude extracts. Dhaka Univ J Sci. 2012;60(1):11-4. doi: 10.3329/dujs. v60i1.10328.

20. Sukito A, Tachibana S. Isolation of hyperoside and isoquercitrin from Camellia sasanqua as antioxidant agents. Pak J Biol Sci. 2014;17(8):999-1006. doi: 10.3923/ pjbs.2014.999.1006.

21. Wolbiś M, Nowak S, Kicel A. Polyphenolic compounds in Scopolia caucasica Kolesn. ex Kreyer (Solanaceae). Acta Pol Pharm. 2007;64(3):241-6.

22. Nath LR, Gorantla JN, Joseph SM, Antony J, Thankachan S, Menon DB, et al. Kaempferide, the most active among the four flavonoids isolated and characterized from Chromolaena odorata, induces apoptosis in cervical cancer cells while being pharmacologically safe. RSC Advances. 2015;5(122):10091222. doi: 10.1039/c5ra19199h.

23. Ibrahim LF, El-Senousy WM, Hawas UW. NMR spectral analysis of flavonoids from Chrysanthemum coronarium. Chem Nat Compd. 2007;43(6):659-62. doi: 10.1007/s10600007-0222-y.

24. Tolonen A, Joutsamo T, Mattlla S, Kämäräinen T, Jalonen J. Identification of isomeric dicaffeoylquinic acids from Eleutherococcus senticosus using HPLC-ESI/TOF/MS and 1H-NMR methods. Phytochem Anal. 2002;13(6):316-28. doi: $10.1002 /$ pca. 663 .

25. Samra RM, Soliman AF, Zaki AA, Ashour A, Al-Karmalawy 
AA, Hassan MA, et al. Bioassay-guided isolation of a new cytotoxic ceramide from Cyperus rotundus L. S Afr J Bot. 2021;139:210-6. doi: 10.1016/j.sajb.2021.02.007.

26. Hatzivassiliou G, Song K, Yen I, Brandhuber BJ, Anderson DJ, Alvarado R, et al. RAF inhibitors prime wild-type RAF to activate the MAPK pathway and enhance growth. Nature. 2010;464(7287):431-5. doi: 10.1038/nature08833.

27. Rastelli G, Rosenfeld R, Reid R, Santi DV. Molecular modeling and crystal structure of ERK2-hypothemycin complexes. J Struct Biol. 2008;164(1):18-23. doi: 10.1016/j. jsb.2008.05.002.

28. Zaki AA, Al-Karmalawy AA, El-Amier YA, Ashour A. Molecular docking reveals the potential of Cleome amblyocarpa isolated compounds to inhibit COVID-19 virus main protease. New J Chem. 2020;44(39):16752-8. doi: 10.1039/d0nj03611k.

29. Al-Karmalawy AA, Khattab M. Molecular modelling of mebendazole polymorphs as a potential colchicine binding site inhibitor. New J Chem. 2020;44(33):13990-6. doi: 10.1039/D0NJ02844D.

30. Khalil H, Abd El Maksoud AI, Roshdey T, El-Masry S. Guava flavonoid glycosides prevent influenza A virus infection via rescue of P53 activity. J Med Virol. 2019;91(1):45-55. doi: 10.1002/jmv.25295.

31. Khalil H, El Malah T, El Maksoud AIA, El Halfawy I, El Rashedy AA, El Hefnawy M. Identification of novel and efficacious chemical compounds that disturb influenza A virus entry in vitro. Front Cell Infect Microbiol. 2017;7:304. doi: $\quad 10.3389 /$ fcimb.2017.00304.

32. Rao X, Huang X, Zhou Z, Lin X. An improvement of the $2^{\wedge}(-$ delta delta CT) method for quantitative real-time polymerase chain reaction data analysis. Biostat Bioinforma Biomath. 2013;3(3):71-85.

33. Li JR, Liu J, He DH, Xu HX, Ding LS, Bao WK, et al. Three new phenolic compounds from the leaves of Rosa sericea. Fitoterapia. 2013;84:332-7. doi: 10.1016/j.fitote.2012.12.019.

34. Zhang Y, Wang D, Yang L, Zhou D, Zhang J. Purification and characterization of flavonoids from the leaves of Zanthoxylum bungeanum and correlation between their structure and antioxidant activity. PLoS One. 2014;9(8):e105725. doi: 10.1371/journal.pone.0105725.

35. Zhang T, Liu H, Liu XT, Xu DR, Chen XQ, Wang Q. Qualitative and quantitative analysis of steroidal saponins in crude extracts from Paris polyphylla var. yunnanensis and $P$. polyphylla var. chinensis by high performance liquid chromatography coupled with mass spectrometry. J Pharm Biomed Anal. 2010;51(1):114-24. doi: 10.1016/j. jpba.2009.08.020.

36. Horai H, Arita M, Kanaya S, Nihei Y, Ikeda T, Suwa K, et al. MassBank: a public repository for sharing mass spectral data for life sciences. J Mass Spectrom. 2010;45(7):703-14. doi: 10.1002/jms. 1777 .

37. Justesen U. Negative atmospheric pressure chemical ionisation low-energy collision activation mass spectrometry for the characterisation of flavonoids in extracts of fresh herbs. J Chromatogr A. 2000;902(2):369-79. doi: 10.1016/ s0021-9673(00)00861-x.

38. Sánchez-Rabaneda F, Jáuregui O, Lamuela-Raventós RM, Viladomat F, Bastida J, Codina C. Qualitative analysis of phenolic compounds in apple pomace using liquid chromatography coupled to mass spectrometry in tandem mode. Rapid Commun Mass Spectrom. 2004;18(5):553-63. doi: $10.1002 / \mathrm{rcm} .1370$.
39. Ben Said R, Hamed AI, Mahalel UA, Al-Ayed AS, Kowalczyk M, Moldoch J, et al. Tentative characterization of polyphenolic compounds in the male flowers of phoenix dactylifera by liquid chromatography coupled with mass spectrometry and DFT. Int J Mol Sci. 2017;18(3):512. doi: 10.3390/ijms18030512.

40. Jaiswal R, Kiprotich J, Kuhnert N. Determination of the hydroxycinnamate profile of 12 members of the Asteraceae family. Phytochemistry. 2011;72(8):781-90. doi: 10.1016/j. phytochem.2011.02.027.

41. Luo C, Wang X, Gao G, Wang L, Li Y, Sun C. Identification and quantification of free, conjugate and total phenolic compounds in leaves of 20 sweetpotato cultivars by HPLC-DAD and HPLC-ESI-MS/MS. Food Chem. 2013;141(3):2697-706. doi: 10.1016/j.foodchem.2013.05.009.

42. Llorent-Martínez EJ, Gouveia S, Castilho PC. Analysis of phenolic compounds in leaves from endemic trees from Madeira Island. A contribution to the chemotaxonomy of Laurisilva forest species. Ind Crop Prod. 2015;64:135-51. doi: 10.1016/j.indcrop.2014.10.068.

43. Benayad Z, Gómez-Cordovés C, Es-Safi NE. Characterization of flavonoid glycosides from fenugreek (Trigonella foenumgraecum) crude seeds by HPLC-DAD-ESI/MS analysis. Int J Mol Sci. 2014;15(11):20668-85. doi: 10.3390/ijms151120668.

44. Spínola V, Pinto J, Castilho PC. Identification and quantification of phenolic compounds of selected fruits from Madeira Island by HPLC-DAD-ESI-MSn and screening for their antioxidant activity. Food Chem. 2015;173:14-30. doi: 10.1016/j.foodchem.2014.09.163.

45. Solorzano ER, Di Gangi IM, Roverso M, Favaro G, Bogialli S, Pastore P. Low level of allergens in the Argentinean plant Zuccagnia punctata Cav.: screening and quality control of North-Western Propolis using an LC-DAD-QTOF system. Appl Sci. 2019;9(17):3546. doi: 10.3390/app9173546.

46. Jaiswal R, Sovdat T, Vivan F, Kuhnert N. Profiling and characterization by LC-MSn of the chlorogenic acids and hydroxycinnamoylshikimate esters in maté (Ilex paraguariensis). J Agric Food Chem. 2010;58(9):5471-84. doi: $10.1021 /$ jf $904537 \mathrm{z}$.

47. ChoJY, Kim JY,Lee YG, LeeHJ, Shim HJ,LeeJH, et al. Four new dicaffeoylquinic acid derivatives from glasswort (Salicornia herbacea L.) and their antioxidative activity. Molecules. 2016;21(8):1097. doi: 10.3390/molecules21081097.

48. Zerback R, Bokel M, Geiger H, Hess D. A kaempferol 3-glucosylgalactoside and further flavonoids from pollen of Petunia hybrida. Phytochemistry. 1989;28(3):897-9. doi: 10.1016/0031-9422(89)80139-6.

49. Simirgiotis MJ, Benites J, Areche C, Sepúlveda B. Antioxidant capacities and analysis of phenolic compounds in three endemic Nolana species by HPLC-PDA-ESI-MS. Molecules. 2015;20(6):11490-507. doi: 10.3390/molecules200611490.

50. Llorach R, Gil-Izquierdo A, Ferreres F, Tomás-Barberán FA. HPLC-DAD-MS/MS ESI characterization of unusual highly glycosylated acylated flavonoids from cauliflower (Brassica oleracea L. var. botrytis) agroindustrial byproducts. J Agric Food Chem. 2003;51(13):3895-9. doi: 10.1021/jf030077h.

51. Schmeda-Hirschmann G, Quispe C, González B. Phenolic profiling of the South American "Baylahuen" tea (Haplopappus spp., Asteraceae) by HPLC-DADESI-MS. Molecules. 2015;20(1):913-28. doi: 10.3390/ molecules20010913.

52. Negri G, Barreto LM, Sper FL, de Carvalho C, da Graça Ribeiro Campos M. Phytochemical analysis and botanical 
origin of Apis mellifera bee pollen from the municipality of Canavieiras, Bahia State, Brazil. Braz J Food Technol. 2018;21: e2016176. doi: 10.1590/1981-6723.17616.

53. Rak G, Fodor P, Abrankó L. Three-step HPLC-ESI-MS/MS procedure for screening and identifying non-target flavonoid derivatives. Int J Mass Spectrom. 2010;290(1):32-8. doi: 10.1016/j.ijms.2009.11.008.

54. Jaiswal R, Kuhnert N. Identification and characterization of two new derivatives of chlorogenic acids in Arnica (Arnica montana L.) flowers by high-performance liquid chromatography/tandem mass spectrometry. J Agric Food Chem. 2011;59(8):4033-9. doi: 10.1021/jf103545k.

55. Abdel-Hady H, El-Sayed MM, Abdel-Gawad MM, ElWakil EA, Abdel-Hameed ES, Abdel-Lateef EE. LC-ESI-MS analysis, antitumor and antioxidant activities of methanolic extract of Egyptian Allium kurrat. J Appl Pharm Sci. 2018;8(7):85-92.

56. Pasini F, Verardo V, Caboni MF, D’Antuono LF. Determination of glucosinolates and phenolic compounds in rocket salad by HPLC-DAD-MS: evaluation of Eruca sativa Mill. and Diplotaxis tenuifolia L. genetic resources. Food Chemistry. 2012;133(3):1025-33. doi: 10.1016/j.foodchem.2012.01.021.

57. Kao TH, Huang SC, Inbaraj BS, Chen BH. Determination of flavonoids and saponins in Gynostemma pentaphyllum (Thunb.) Makino by liquid chromatography-mass spectrometry. Anal Chim Acta. 2008;626(2):200-11. doi: 10.1016/j.aca.2008.07.049.

58. Harbaum B, Hubbermann EM, Wolff C, Herges R, Zhu Z, Schwarz K. Identification of flavonoids and hydroxycinnamic acids in pak choi varieties (Brassica campestris L. ssp. chinensis var. communis) by HPLC-ESI-MSn and NMR and their quantification by HPLC-DAD. J Agric Food Chem. 2007;55(20):8251-60. doi: 10.1021/jf071314+.

59. Brito A, Ramirez JE, Areche C, Sepúlveda B, Simirgiotis MJ. HPLC-UV-MS profiles of phenolic compounds and antioxidant activity of fruits from three citrus species consumed in Northern Chile. Molecules. 2014;19(11):1740021. doi: 10.3390/molecules191117400.

60. Mehrbod P, Abdalla MA, Fotouhi F, Heidarzadeh M, Aro AO, Eloff JN, et al. Immunomodulatory properties of quercetin3-O-a-L-rhamnopyranoside from Rapanea melanophloeos against influenza a virus. BMC Complement Altern Med. 2018;18(1):184. doi: 10.1186/s12906-018-2246-1.

61. Jang GH, Kim HW, Lee MK, Jeong SY, Bak AR, Lee DJ, et al. Characterization and quantification of flavonoid glycosides in the Prunus genus by UPLC-DAD-QTOF/MS. Saudi J Biol Sci. 2018;25(8):1622-31. doi: 10.1016/j.sjbs.2016.08.001.

62. Weckerle B, Michel K, Balázs B, Schreier P, Tóth G. Quercetin 3,3'4'-tri-O-beta-D-glucopyranosides from leaves of Eruca sativa (Mill.). Phytochemistry. 2001;57(4):547-51. doi: 10.1016/s0031-9422(01)00059-0.

63. Mokrani A, Cluzet S, Madani K, Pakina E, Gadzhikurbanov A, Mesnil M, et al. HPLC-DAD-MS/MS profiling of phenolics from different varieties of peach leaves and evaluation of their antioxidant activity: a comparative study. Int J Mass Spectrom. 2019;445:116192. doi: 10.1016/j. ijms.2019.116192.

64. El-Sadek A, Balah M, Romani A, Francesca Ieri F,
Vignolini P, Salem E, et al. Allelopathic potential of quinoa (Chenopodium quinoa Willd.) genotypes on the germination and initial development of some weeds and crops. Egypt J Desert Res. 2017;67(1):25-45. doi: 10.21608/ejdr.2017.5843.

65. Guo S, Duan JA, Tang Y, Qian Y, Zhao J, Qian D, et al. Simultaneous qualitative and quantitative analysis of triterpenic acids, saponins and flavonoids in the leaves of two Ziziphus species by HPLC-PDA-MS/ELSD. J Pharm Biomed Anal. 2011;56(2):264-70. doi: 10.1016/j.jpba.2011.05.025.

66. Oszmiański J, Kolniak-Ostek J, Wojdyło A. Application of ultra performance liquid chromatography-photodiode detector-quadrupole/time of flight-mass spectrometry (UPLC-PDA-Q/TOF-MS) method for the characterization of phenolic compounds of Lepidium sativum L. sprouts. Eur Food Res Technol. 2013;236(4):699-706. doi: 10.1007/ s00217-013-1925-x.

67. Zanutto FV, Boldrin PK, Varanda EA, Souza SF, Sano PT, Vilegas W, et al. Characterization of flavonoids and naphthopyranones in methanol extracts of Paepalanthus chiquitensis Herzog by HPLC-ESI-IT-MSn and their mutagenic activity. Molecules. 2013;18(1):244-62. doi: 10.3390/molecules 18010244 .

68. Hsu BY, Lin SW, Inbaraj BS, Chen BH. Simultaneous determination of phenolic acids and flavonoids in Chenopodium formosanum Koidz. (djulis) by HPLC-DADESI-MS/MS. J Pharm Biomed Anal. 2017;132:109-16. doi: 10.1016/j.jpba.2016.09.027.

69. Regos I. Chemical Characterisation of Low Molecular Weight Phenolic Compounds from the Forage Legume Sainfoin (Onobrychis viciifolia) [dissertation]. Munich: Technische Universität München; 2014.

70. Kicel A, Wolbiś M. Study on the phenolic constituents of the flowers and leaves of Trifolium repens L. Nat Prod Res. 2012;26(21):2050-4. doi: 10.1080/14786419.2011.637217.

71. Handoussa H, Hanafi R, Eddiasty I, El-Gendy M, El Khatib $A$, Linscheid $\mathrm{M}$, et al. Anti-inflammatory and cytotoxic activities of dietary phenolics isolated from Corchorus olitorius and Vitis vinifera. J Funct Foods. 2013;5(3):1204-16. doi: 10.1016/j.jff.2013.04.003.

72. Ibrahim RM, El-Halawany AM, Saleh DO, El Naggar EM, El-Shabrawy AE, El-Hawary SS. HPLC-DAD-MS/MS profiling of phenolics from Securigera securidaca flowers and its anti-hyperglycemic and anti-hyperlipidemic activities. Rev Bras Farmacogn. 2015;25(2):134-41. doi: 10.1016/j. bjp.2015.02.008.

73. Adnane L, Trail PA, Taylor I, Wilhelm SM. Sorafenib (BAY 43-9006, Nexavar), a dual-action inhibitor that targets RAF/ MEK/ERK pathway in tumor cells and tyrosine kinases VEGFR/PDGFR in tumor vasculature. Methods Enzymol. 2006;407:597-612. doi: 10.1016/s0076-6879(05)07047-3.

74. Zhang Y, Li G, Liu X, Song Y, Xie J, Li G, et al. Sorafenib inhibited cell growth through the MEK/ERK signaling pathway in acute promyelocytic leukemia cells. Oncol Lett. 2018;15(4):5620-6. doi: 10.3892/ol.2018.8010.

75. Wu J, Dent P, Jelinek T, Wolfman A, Weber MJ, Sturgill TW. Inhibition of the EGF-activated MAP kinase signaling pathway by adenosine 3', ''-monophosphate. Science. 1993;262(5136):1065-9. doi: 10.1126/science.7694366. 